\title{
Definition of a general implicit orthotropic yield criterion
}

\author{
Sergio Oller ${ }^{\mathrm{a}, *}$, Eduardo Car ${ }^{\mathrm{a}}$, Jacob Lubliner ${ }^{\mathrm{b}}$ \\ ${ }^{a}$ Departamento de Resistencia de Materiales y Estructuras en la Ingeniería, Universidad Politécnica de Cataluña, \\ Jordi Girona 1-3, Módulo C1 Campus Norte UPC, Barcelona 08034, Spain \\ ${ }^{\mathrm{b}}$ Department of Civil and Environmental Engineering, University of California at Berkeley, Berkeley, CA 94720, USA
}

Received 20 September 2001; received in revised form 29 October 2002

\begin{abstract}
The definition of an orthotropic yield criterion presents a serious challenge in the formulation of constitutive models based on such theories as elastoplasticity, viscoplasticity, damage, etc. The need to model the behavior of a real orthotropic material requires the formulation of orthotropic yield criteria, and these may be difficult to obtain. For metals, orthotropic yield functions have been formulated by Hill [Proc. Roy. Soc. Lon. Ser. A 193 (1948) 281; J. Mech. Phys. Solids 38(3) (1990) 200], Barlat [Int. J. Plasticity 5 (1989) 51; 7 (1991) 693], Chu [NUMISHEET 93 (1993) 199] and Dutko et al. [Comput. Methods Appl. Mech. Engrg. 109 (1993) 73], but in many cases these functions do not describe the true behavior of the metal. The situation is worse when one attempts to represent a nonmetal such as a polymer, ceramic or composite.

In this paper, we present a general definition of an explicit orthotropic yield criterion together with a general method for defining implicit orthotropic yield functions. The latter formulation is based on the transformed-tensor method, whose principal advantage lies in the possibility of adjusting an arbitrary isotropic yield criterion to the behavior of an anisotropic material. As example we choose the adjustment to the Hill, Hoffman [J. Comp. Materials 1 (1967) 200] and Tsai-Wu [J. Comp. Materials 5 (1971) 58] criteria, but these particular cases serve to establish the methodology for achieving the desired function adjustment for any other well-known criterion or experimental set of data obtained from laboratory.
\end{abstract}

(c) 2002 Elsevier Science B.V. All rights reserved.

\section{Introduction}

The work presented in what follows has as its objective the establishment of a tool for formulating orthotropic yield functions from isotropic ones with the help of the experimental knowledge of the material and of the transformed-tensor method [3,4,21]. The advantage of this procedure is due to the fact that implicitly convex orthotropic functions are obtained from well-established isotropic ones, such as those of von Mises, Drucker-Prager, Tresca, Mohr-Coulomb, etc. [14,15]. This working strategy

\footnotetext{
${ }^{*}$ Corresponding author.

E-mail addresses: sergio.oller@upc.es (S. Oller), car@cimne.upc.es (E. Car), lubliner@ce.berkeley.edu (J. Lubliner).
} 
allows the establishment of the implicit orthotropic yield criterion in the "real" anisotropic space by using the isotropic one formulated on the "ideal" (fictitious) isotropic space, with all the advantages implied by this.

Part of this work is based on previous work by two of the authors $[7,16,17,19]$, showing the foundations of the transformed-tensor method; here we add the generalization of this concept and the adjustment necessary for approximating the desired behavior.

The work shown here is as follows: first, a general presentation of the yield criteria, in which the relations that allow the analytical adjustment of a general criterion to various particular cases are determined, as well as the method for establishing these formulations by means of the transformed-tensor method. Next, the definition of the transformation tensor that allows transforms the isotropic von Mises criterion into the orthotropic one of Hill and the isotropic Drucker-Prager criterion into the orthotropic ones of Tsai-Wu and Hoffman are presented. Finally, a numerical adjustment procedure for performing the transformation is established.

\section{General definition of an isotropic yield criterion}

In this section we propose a general form of an isotropic yield criterion in which the yield stress in simple tension, $f^{t}$, is different from the one in compression, $f^{c}$. It is
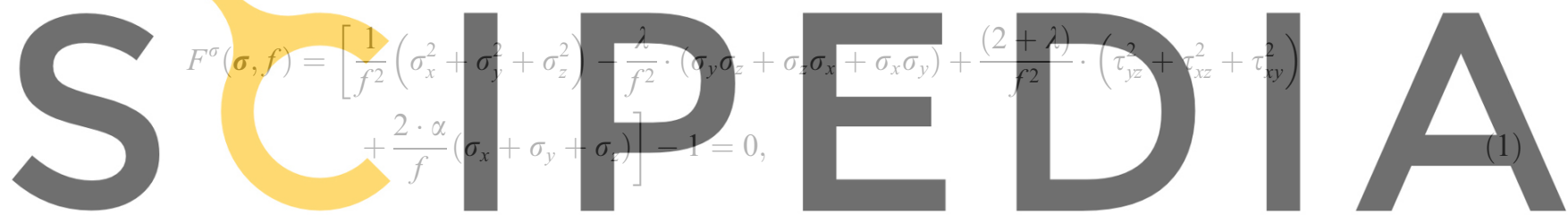

where $f=\sqrt{f^{\mathrm{t}} f^{\mathrm{c}}}$ is the equivalent yield stress, $\alpha=\frac{1}{2}\left[\left(f^{\mathrm{c}} / f^{\mathrm{t}}\right)-\left(f^{\mathrm{t}} / f^{\mathrm{c}}\right)\right]=\frac{1}{2 f^{2}}\left(\left(f^{\mathrm{c}}\right)^{2}-\left(f^{\mathrm{t}}\right)^{2}\right)$ is an adjust-

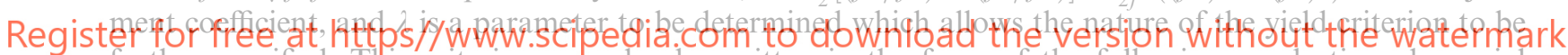
further specified. This criterion can also be written in the form of the following quadratic polynomial expression

$$
F^{\sigma}(\boldsymbol{\sigma}, f)=\left[\boldsymbol{\sigma}^{\mathrm{T}} \cdot \mathbf{P}_{2}^{\text {iso }} \cdot \boldsymbol{\sigma}+\mathbf{P}_{1}^{\text {iso }} \cdot \boldsymbol{\sigma}\right]-1=0
$$

with

$$
\mathbf{P}_{2}^{\text {iso }}=\frac{1}{f^{2}} \cdot\left[\begin{array}{cccccc}
1 & -\frac{\lambda}{2} & -\frac{\lambda}{2} & 0 & 0 & 0 \\
-\frac{\lambda}{2} & 1 & -\frac{\lambda}{2} & 0 & 0 & 0 \\
\lambda & -\frac{\lambda}{2} & 1 & 0 & 0 & 0 \\
0 & 0 & 0 & 2+\lambda & 0 & 0 \\
0 & 0 & 0 & 0 & 2+\lambda & 0 \\
0 & 0 & 0 & 0 & 0 & 2+\lambda
\end{array}\right], \quad \mathbf{P}_{1}^{\text {iso }}=\left\{\begin{array}{c}
\frac{2 \alpha}{f} \\
\frac{2 \alpha}{f} \\
\frac{2 \alpha}{f} \\
0 \\
0 \\
0
\end{array}\right\} .
$$

Oriented to the numerical approach formulated in this paper, Eq. (2) also can be written in the following more compact form

$$
F^{\sigma}(\boldsymbol{\sigma}, f)=\left[\boldsymbol{\sigma}^{\mathrm{T}} \cdot \mathbb{P}^{\mathrm{iso}}(\boldsymbol{\sigma}) \cdot \boldsymbol{\sigma}\right]-1=0
$$


with

$$
\mathbb{P}^{\text {iso }}(\boldsymbol{\sigma})=\frac{1}{f^{2}} \cdot\left[\begin{array}{cccccc}
1+\frac{2 \alpha f}{\sigma_{x}} & -\frac{\lambda}{2} & -\frac{\lambda}{2} & 0 & 0 & 0 \\
-\frac{\lambda}{2} & 1+\frac{2 \alpha f}{\sigma_{y}} & -\frac{\lambda}{2} & 0 & 0 & 0 \\
-\frac{\lambda}{2} & -\frac{\lambda}{2} & 1+\frac{2 \alpha f}{\sigma_{z}} & 0 & 0 & 0 \\
0 & 0 & 0 & 2+\lambda & 0 & 0 \\
0 & 0 & 0 & 0 & 2+\lambda & 0 \\
0 & 0 & 0 & 0 & 0 & 2+\lambda
\end{array}\right],
$$

where $\boldsymbol{\sigma}^{\mathrm{T}}=\left\{\sigma_{x}, \sigma_{y}, \sigma_{z}, \tau_{x y}, \tau_{y z}, \tau_{x z}\right\}$ is a column matrix representing the symmetric part of the stress tensor, and $\mathbf{P}_{1}^{\text {iso }}, \mathbf{P}_{2}^{\text {iso }}$ and $\mathbb{P}^{\text {iso }}(\boldsymbol{\sigma})$ are the matrices that allow the canonical form expressed in Eq. (1) to be recovered. Note that $\mathbb{P}^{\text {iso }}(\sigma)$ depends on the stress if $\alpha \neq 0$.

The criterion thus formulated can describe the elastic-limit behavior of various material types, from geomaterials to metals, depending on an appropriate choice of the parameters $\alpha$ and $\lambda$. In the hypothetical case of a metal, in which the relations $f_{\mathrm{c}} \equiv f_{\mathrm{t}} \equiv f \Rightarrow \alpha=0$ and $\lambda=1$ are satisfied, one recovers the classical von Mises criterion [15] expressed in the form

$F^{\sigma}(\sigma, f)=\left[\frac{1}{f^{2}}\left(\sigma_{x}^{2}+\sigma_{y}^{2}+\sigma_{z}^{2}\right)-\frac{1}{f^{2}}\left(\sigma_{y} \sigma_{z}+\sigma_{z} \sigma_{x}+\sigma_{x} \sigma_{y}\right)+\frac{3}{f^{2}} \cdot\left(\tau_{y z}^{2}+\tau_{x z}^{2}+\tau_{x y}^{2}\right)\right]-1=0$
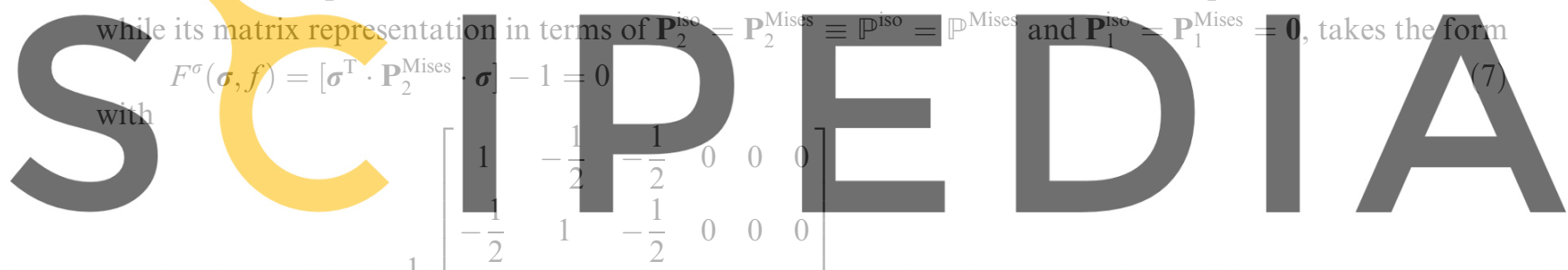

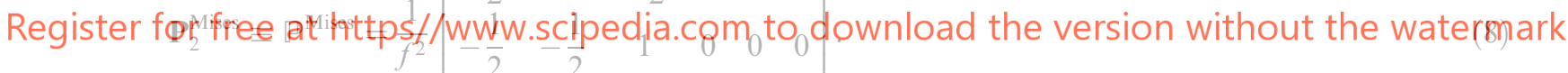

$$
\left[\begin{array}{llllll}
0 & 0 & 0 & 3 & 0 & 0 \\
0 & 0 & 0 & 0 & 3 & 0 \\
0 & 0 & 0 & 0 & 0 & 3
\end{array}\right]
$$

The Mises-Schleicher criterion [15] is recovered by assuming that $f^{\mathrm{c}}>f^{\mathrm{t}} \Rightarrow \alpha>0$ and $\lambda=1$, while the Drucker-Prager criterion [15] is obtained by rearranging Eq. (1), letting $f^{\mathrm{c}} \neq f^{\mathrm{t}}$ and $\lambda=1+3 \alpha^{2}$, that is,

$$
\begin{aligned}
F^{\sigma}(\boldsymbol{\sigma}, f)= & \sqrt{\left(\sigma_{x}^{2}+\sigma_{y}^{2}+\sigma_{z}^{2}\right)-\left(\sigma_{y} \sigma_{z}+\sigma_{z} \sigma_{x}+\sigma_{x} \sigma_{y}\right)+3 \cdot\left(\tau_{y z}^{2}+\tau_{x z}^{2}+\tau_{x y}^{2}\right)} \\
& +\frac{\alpha}{1+\alpha^{2}}\left(\sigma_{x}+\sigma_{y}+\sigma_{z}\right)-\frac{f}{\sqrt{1+\alpha^{2}}}=0 .
\end{aligned}
$$

This way of formulating the yield criteria allows us to add a further degree of generality and eventually to define orthotropic yield criteria.

\section{Explicit general definition of an orthotropic yield criterion}

Just as Hill [11,12], Tsai and Wu [23] and Hoffman [13] formulated their yield criteria for orthotropic materials as a generalization of the isotropic criterion, Eq. (1) can now be generalized into an explicit orthotropic formulation as follows: 


$$
\begin{aligned}
F^{\sigma}(\boldsymbol{\sigma}, \mathbf{f})= & {\left[\left(\frac{\sigma_{x}^{2}}{f_{x}^{\mathrm{t}} f_{x}^{\mathrm{c}}}+\frac{\sigma_{y}^{2}}{f_{y}^{\mathrm{t}} f_{y}^{\mathrm{c}}}+\frac{\sigma_{z}^{2}}{f_{z}^{\mathrm{t}} f_{z}^{\mathrm{c}}}\right)-\lambda_{1}\left(\frac{\sigma_{y} \sigma_{z}}{\sqrt{f_{y}^{\mathrm{t}} f_{y}^{\mathrm{c}} f_{z}^{\mathrm{t}} f_{z}^{\mathrm{c}}}}\right)-\lambda_{2}\left(\frac{\sigma_{z} \sigma_{x}}{\sqrt{f_{z}^{\mathrm{t}} f_{z}^{\mathrm{c}} f_{x}^{\mathrm{t}} f_{x}^{\mathrm{c}}}}\right)-\lambda_{3}\left(\frac{\sigma_{x} \sigma_{y}}{\sqrt{f_{x}^{\mathrm{t}} f_{x}^{\mathrm{c}} f_{y}^{\mathrm{t}} f_{y}^{\mathrm{c}}}}\right)\right.} \\
& \left.+\left(\frac{\tau_{y z}^{2}}{f_{y z}^{2}}+\frac{\tau_{x z}^{2}}{f_{x z}^{2}}+\frac{\tau_{x y}^{2}}{f_{x y}^{2}}\right)+2 \cdot\left(\alpha_{x} \frac{\sigma_{x}}{\sqrt{f_{x}^{\mathrm{t}} f_{x}^{\mathrm{c}}}}+\alpha_{y} \frac{\sigma_{y}}{\sqrt{f_{y}^{\mathrm{t}} f_{y}^{\mathrm{c}}}}+\alpha_{z} \frac{\sigma_{z}}{\sqrt{f_{z}^{\mathrm{t}} f_{z}^{\mathrm{c}}}}\right)\right]-1=0,
\end{aligned}
$$

where $x, y, z$ are the principal axes of orthotropy, while $\mathbf{f}=\left\{f_{x}, f_{y}, f_{z}, f_{x y}, f_{y z}, f_{x z}\right\}=\left\{\sqrt{f_{x}^{\mathrm{t}} f_{x}^{\mathrm{c}}}, \sqrt{f_{y}^{\mathrm{t}} f_{y}^{\mathrm{c}}}\right.$, $\left.\sqrt{f_{z}^{\mathrm{t}} f_{z}^{\mathrm{c}}}, f_{x y}, f_{y z}, f_{x z}\right\}$ is the row matrix of yield stresses (axial and shear) with respect to these axes, and $\alpha_{i}=\frac{1}{2}\left[\left(f_{i}^{\mathrm{c}} / f_{i}^{\mathrm{t}}\right)^{m}-\left(f_{i}^{\mathrm{t}} / f_{i}^{\mathrm{c}}\right)^{m}\right] \forall i=x, y, z$. The matrix form of Eq. (10) can be written as

$$
F^{\sigma}(\boldsymbol{\sigma}, \mathbf{f})=\left[\boldsymbol{\sigma}^{\mathrm{T}} \cdot \mathbf{P}_{2}^{\text {ort }} \cdot \boldsymbol{\sigma}+\mathbf{P}_{1}^{\text {ort }} \cdot \boldsymbol{\sigma}\right]-1=0
$$

with
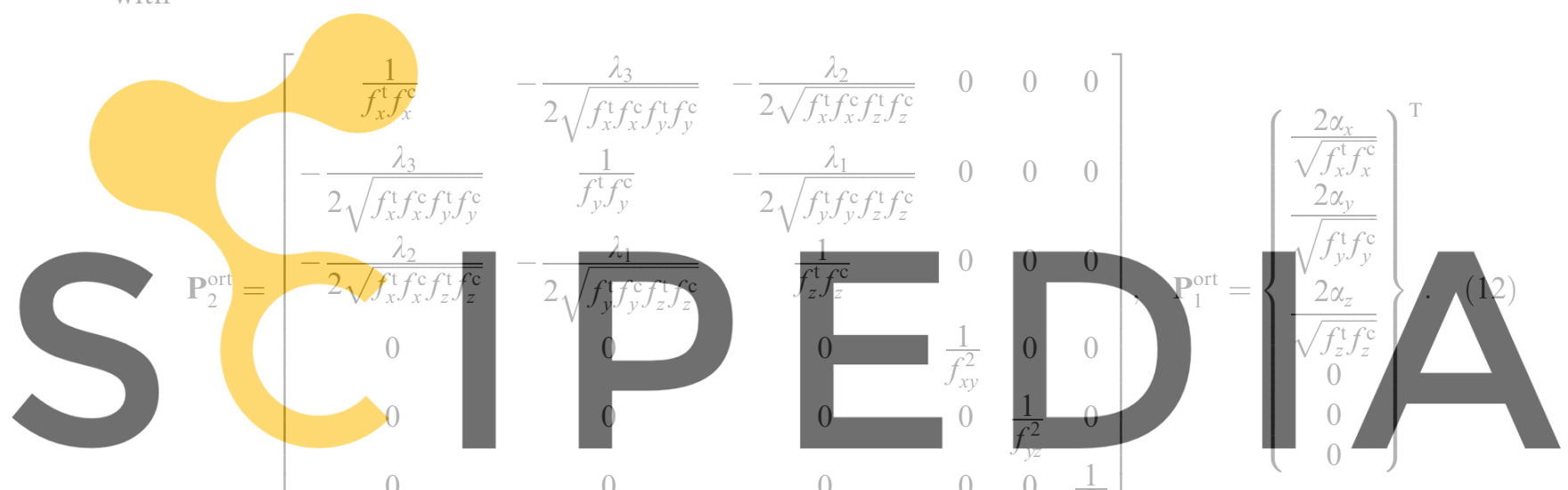

Register for free lat https//www.scipedia.com to download the version without the watermark

Eq. (11) can also be written in the following compact form

$$
F^{\sigma}(\boldsymbol{\sigma}, \mathbf{f})=\left[\boldsymbol{\sigma}^{\mathrm{T}} \cdot \mathbb{P}^{\text {ort }}(\boldsymbol{\sigma}) \cdot \boldsymbol{\sigma}\right]-1=0
$$

with

$$
\mathbb{P}^{\text {ort }}(\boldsymbol{\sigma})=\left[\begin{array}{cccccc}
\frac{1}{f_{x}^{\mathrm{t}} f_{x}^{\mathrm{c}}}+\frac{2 \alpha_{x}}{\sigma_{x} \sqrt{f_{x}^{\mathrm{t}} f_{x}^{\mathrm{c}}}} & -\frac{\lambda_{3}}{2 \sqrt{f_{x}^{\mathrm{t}} f_{x}^{\mathrm{c}} f_{y}^{\mathrm{t}} f_{y}^{\mathrm{c}}}}-\frac{\lambda_{2}}{2 \sqrt{f_{x}^{\mathrm{t}} f_{x}^{\mathrm{c}} f_{z}^{\mathrm{t}} f_{z}^{\mathrm{c}}}} & 0 & 0 & 0 \\
-\frac{\lambda_{3}}{2 \sqrt{f_{x}^{\mathrm{t}} f_{x}^{\mathrm{c}} f_{y}^{\mathrm{t}} f_{y}^{\mathrm{c}}}} & \frac{1}{f_{y}^{\mathrm{t}} f_{y}^{\mathrm{c}}}+\frac{2 \alpha_{x}}{\sigma_{y} \sqrt{f_{y}^{\mathrm{t}} f_{y}^{\mathrm{c}}}} & -\frac{\lambda_{1}}{2 \sqrt{f_{y}^{\mathrm{t}} f_{y}^{\mathrm{c}} f_{z}^{\mathrm{t}} f_{z}^{\mathrm{c}}}} & 0 & 0 & 0 \\
-\frac{\lambda_{2}}{2 \sqrt{f_{x}^{\mathrm{t}} f_{x}^{\mathrm{c}} f_{z}^{\mathrm{t}} f_{z}^{\mathrm{c}}}} & -\frac{\lambda_{1}}{2 \sqrt{f_{y}^{\mathrm{t}} f_{y}^{\mathrm{c}} f_{z}^{\mathrm{t}} f_{z}^{\mathrm{c}}}} & \frac{1}{f_{z}^{\mathrm{t}} f_{z}^{\mathrm{c}}}+\frac{2 \alpha_{x}}{\sigma_{z} \sqrt{f_{z}^{\mathrm{t}} f_{z}^{\mathrm{c}}}} & 0 & 0 & 0 \\
0 & 0 & 0 & \frac{1}{f_{x y}^{2}} & 0 & 0 \\
0 & 0 & 0 & 0 & \frac{1}{f_{y z}^{2}} & 0 \\
0 & 0 & 0 & 0 & 0 & \frac{1}{f_{x z}^{2}}
\end{array}\right] .
$$


As can be seen, Eq. (10) reduces to the isotropic form, Eq. (1), when the following conditions are met:

$$
\begin{aligned}
\lambda_{1} & =\lambda_{2}=\lambda_{3} \equiv \lambda, \quad m=1, \quad f_{x}^{\mathrm{c}}=f_{y}^{\mathrm{c}}=f_{z}^{\mathrm{c}}=f^{\mathrm{c}}, \quad f_{x}^{\mathrm{t}}=f_{y}^{\mathrm{t}}=f_{z}^{\mathrm{t}}=f^{\mathrm{t}}, \quad f=\sqrt{f^{\mathrm{t}} f^{\mathrm{c}}} f_{y z}=f_{x z} \\
& =f_{x y} \equiv f_{\tau}=\frac{f}{\sqrt{2+\lambda}}=\frac{\sqrt{f^{\mathrm{t}} f^{\mathrm{c}}}}{\sqrt{2+\lambda}}, \quad \alpha_{x}=\alpha_{y}=\alpha_{z}=\alpha \equiv \frac{1}{2}\left[\left(\frac{f^{\mathrm{c}}}{f^{\mathrm{t}}}\right)-\left(\frac{f^{\mathrm{t}}}{f^{\mathrm{c}}}\right)\right] .
\end{aligned}
$$

In these expressions the relations between the orthotropic and the isotropic quantities can be readily observed.

The following subsections show the Mises-Hill, Tsai-Wu and Hoffman criteria can be derived as particular forms of the general Eq. (10).

\subsection{Orthotropic Mises-Hill criterion}

The orthotropic Mises-Hill criterion is obtained from Eq. (10) by making the yield stresses in all directions equal in tension and compression, $f_{i} \equiv f_{i}^{\mathrm{c}}=f_{i}^{\mathrm{t}} \forall i=x, y, z$, leading to $\alpha_{x}=\alpha_{y}=\alpha_{z}=0 \Rightarrow \mathbf{P}_{1}^{\text {ort }}=$ $\mathbf{P}_{1}^{\text {Hitl }}=\mathbf{0}$, and furthermore subjecting the $\lambda_{i}$ to the following restriction:

$$
\lambda_{1} \lambda_{2} \lambda_{3}+\lambda_{1}^{2}+\lambda_{2}^{2}+\lambda_{3}^{2}=4
$$

The result is the Mises-Hill criterion

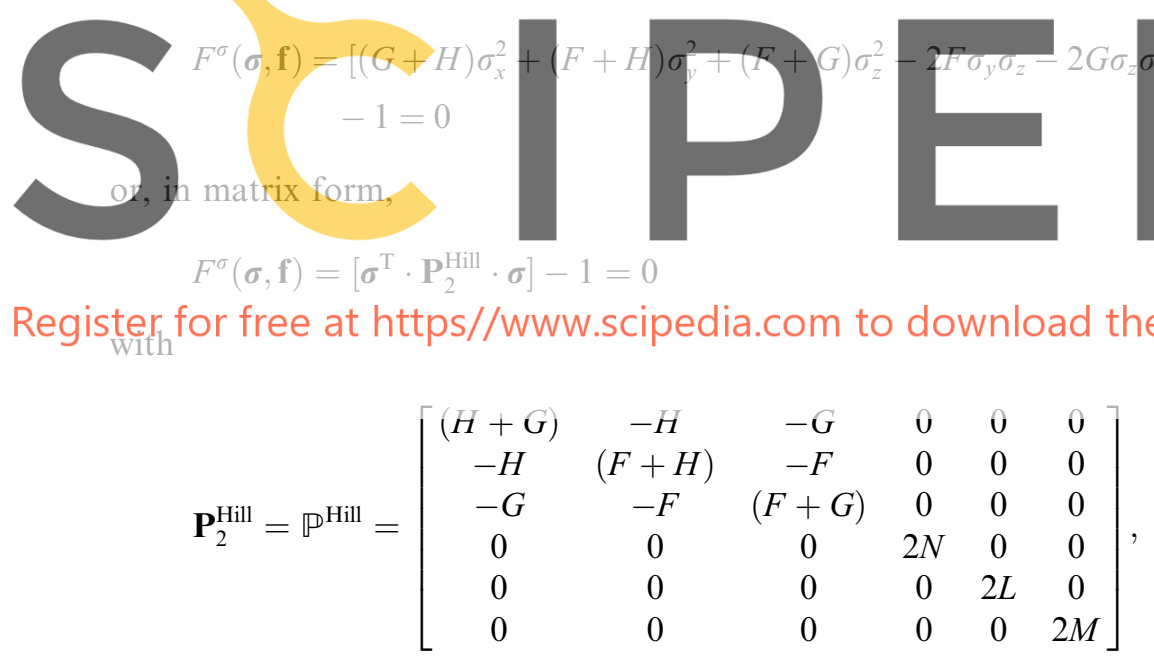

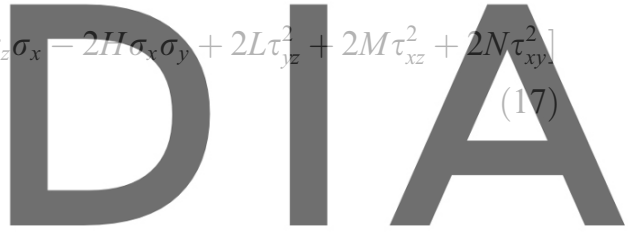

(18)

where $\boldsymbol{\sigma}^{\mathrm{t}}=\left\{\sigma_{x}, \sigma_{y}, \sigma_{z}, \tau_{x y}, \tau_{y z}, \tau_{x z}\right\}$ is the row-matrix representation of the stress tensor and $\mathbf{P}_{2}^{\text {ort }}=$ $\mathbf{P}_{2}^{\text {Hill }}=\mathbb{P}^{\text {Hill }}$ is the square matrix that allows the canonical form expressed in Eq. (17) to be recovered.

Comparing Eqs. (17) and (10), one finds the following relations between the yield stresses and Hill's parameters:

$$
\left\{\begin{array}{lll}
\frac{1}{f_{x}^{2}}=(G+H), & \frac{\lambda_{1}}{f_{y} f_{z}}=2 F, & \frac{1}{f_{y z}^{2}}=2 L \\
\frac{1}{f_{y}^{2}}=(F+H), & \frac{\lambda_{2}}{f_{x} f_{z}}=2 G, & \frac{1}{f_{x z}^{2}}=2 M \\
\frac{1}{f_{z}^{2}}=(F+G), & \frac{\lambda_{3}}{f_{x} f_{y}}=2 H, & \frac{1}{f_{x y}^{2}}=2 N
\end{array}\right.
$$


Upon substituting $F, G, y$ and $H$ as given by the second column of Eq. (20) in the first column, a system of equations for the $\lambda_{i}$ is found, and can be solved with the result

$$
\left\{\begin{array}{l}
\lambda_{1}=\frac{f_{z}^{2} f_{x}^{2}-f_{z}^{2} f_{y}^{2}+f_{y}^{2} f_{x}^{2}}{f_{y} f_{z} f_{x}^{2}} \\
\lambda_{2}=\frac{-f_{z}^{2} f_{x}^{2}+f_{z}^{2} f_{y}^{2}+f_{y}^{2} f_{x}^{2}}{f_{x} f_{y}^{2}} \\
\lambda_{3}=\frac{f_{z}^{2} f_{x}^{2}+f_{z}^{2} f_{y}^{2}-f_{y}^{2} f_{x}^{2}}{f_{y} f_{x} f_{z}^{2}} .
\end{array}\right.
$$

From this result the satisfaction of the conditions required by Eq. (16) can be deduced. Substituting Eq. (21) in (20), we find Hill's parameters in terms of the yield stresses

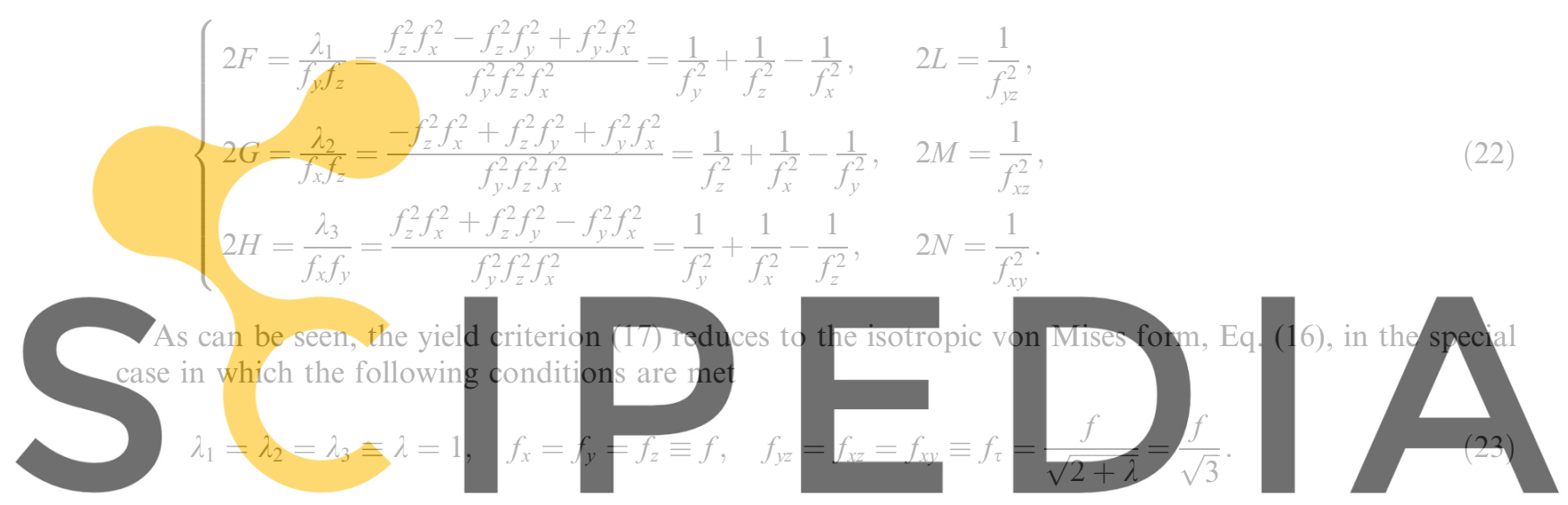

Eq. (10) also reduces to the orthotropic Hill-Schleicher criterion when, in addition to Eq. (16), the or-

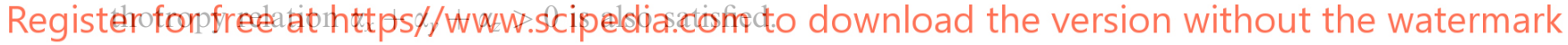

\subsection{Orthotropic Tsai-Wu and Hoffman criteria}

The Hoffman [13] and Tsai-Wu [23] orthotropic yield criteria are represented by a complete polynomial function of second degree (with quadratic, linear and constant terms) in the stresses. They are very useful tools for the failure prediction of the composite materials [9].

The mathematical expression of these yield criteria is also a particular case of the general yield criterion given by Eq. (10), after making $m=1 / 2$ in the definition $\alpha_{i}=\frac{1}{2}\left[\left(f_{i}^{\mathrm{c}} / f_{i}^{\mathrm{t}}\right)^{m}-\left(f_{i}^{\mathrm{t}} / f_{i}^{\mathrm{c}}\right)^{m}\right] \forall i=x, y, z$, and introducing the following assumptions:

- For the Tsai-Wu criterion: $\lambda_{i}=1 \forall i=x, y, z$.

- For the Hoffman criterion:

$$
\left\{\begin{array}{l}
\lambda_{1}=-\frac{\sqrt{f_{y}^{\mathrm{t}} f_{y}^{\mathrm{c}} f_{z}^{\mathrm{t}} f_{z}^{\mathrm{c}}}}{f_{x}^{\mathrm{t}} f_{x}^{\mathrm{c}}}+\frac{f_{z}^{\mathrm{t}} f_{z}^{\mathrm{c}}}{\sqrt{f_{y}^{\mathrm{t}} f_{y}^{\mathrm{c}} f_{z}^{\mathrm{t}} f_{z}^{\mathrm{c}}}}+\frac{f_{y}^{\mathrm{t}} f_{y}^{\mathrm{c}}}{\sqrt{f_{y}^{\mathrm{t}} f_{y}^{\mathrm{c}} f_{z}^{\mathrm{t}} f_{z}^{\mathrm{c}}}}, \\
\lambda_{2}=-\frac{\sqrt{f_{z}^{\mathrm{t}} f_{z}^{\mathrm{c}} f_{x}^{\mathrm{t}} f_{x}^{\mathrm{c}}}}{f_{y}^{\mathrm{t}} f_{y}^{\mathrm{c}}}+\frac{f_{x}^{\mathrm{t}} f_{x}^{\mathrm{c}}}{\sqrt{f_{z}^{\mathrm{t}} f_{z}^{\mathrm{c}} f_{x}^{\mathrm{t}} f_{x}^{\mathrm{c}}}}+\frac{f_{z}^{\mathrm{t}} f_{z}^{\mathrm{c}}}{\sqrt{f_{z}^{\mathrm{t}} f_{z}^{\mathrm{c}} f_{x}^{\mathrm{t}} f_{x}^{\mathrm{c}}}}, \\
\lambda_{3}=-\frac{\sqrt{f_{x}^{\mathrm{t}} f_{x}^{\mathrm{c}} f_{y}^{\mathrm{t}} f_{y}^{\mathrm{c}}}}{f_{z}^{\mathrm{t}} f_{z}^{\mathrm{c}}}+\frac{f_{x}^{\mathrm{t}} f_{x}^{\mathrm{c}}}{\sqrt{f_{x}^{\mathrm{t}} f_{x}^{\mathrm{c}} f_{y}^{\mathrm{t}} f_{y}^{\mathrm{c}}}}+\frac{f_{y}^{\mathrm{t}} f_{y}^{\mathrm{c}}}{\sqrt{f_{x}^{\mathrm{t}} f_{x}^{\mathrm{c}} f_{y}^{\mathrm{t}} f_{y}^{\mathrm{c}}}}
\end{array}\right.
$$


For the Tsai-Wu and Hoffman criteria the following relationship is satisfied in the same way as for the MisesHill criterion

$$
\lambda_{1} \lambda_{2} \lambda_{3}+\lambda_{1}^{2}+\lambda_{2}^{2}+\lambda_{3}^{2}=4
$$

The Tsai-Wu and Hoffman criteria can also be expressed in the following general form:

$$
\begin{aligned}
F^{\sigma}(\sigma, \mathbf{f})= & {\left[(G+H) \sigma_{x}^{2}+(F+H) \sigma_{y}^{2}+(F+G) \sigma_{z}^{2}-2 \tilde{F} \sigma_{y} \sigma_{z}-2 \tilde{G} \sigma_{z} \sigma_{x}-2 \tilde{H} \sigma_{x} \sigma_{y}+2 L \tau_{y z}^{2}+2 M \tau_{x z}^{2}+2 N \tau_{x y}^{2}\right] } \\
& +\left[I \sigma_{x}+J \sigma_{y}+K \sigma_{z}\right]-1=0
\end{aligned}
$$

or

$$
F^{\sigma}(\boldsymbol{\sigma}, \mathbf{f})=[a(\boldsymbol{\sigma})]^{\text {quad }}+[b(\boldsymbol{\sigma})]^{\text {Linear }}+1=0
$$

or, in matrix form,

$$
F^{\sigma}(\sigma, \mathbf{f})=\left[\sigma^{\mathrm{T}} \cdot \mathbf{P}_{2}^{\mathrm{TW}-\mathrm{H}} \cdot \sigma+\mathbf{P}_{1}^{\mathrm{TW}-\mathrm{H}} \cdot \sigma\right]-1=0
$$

with

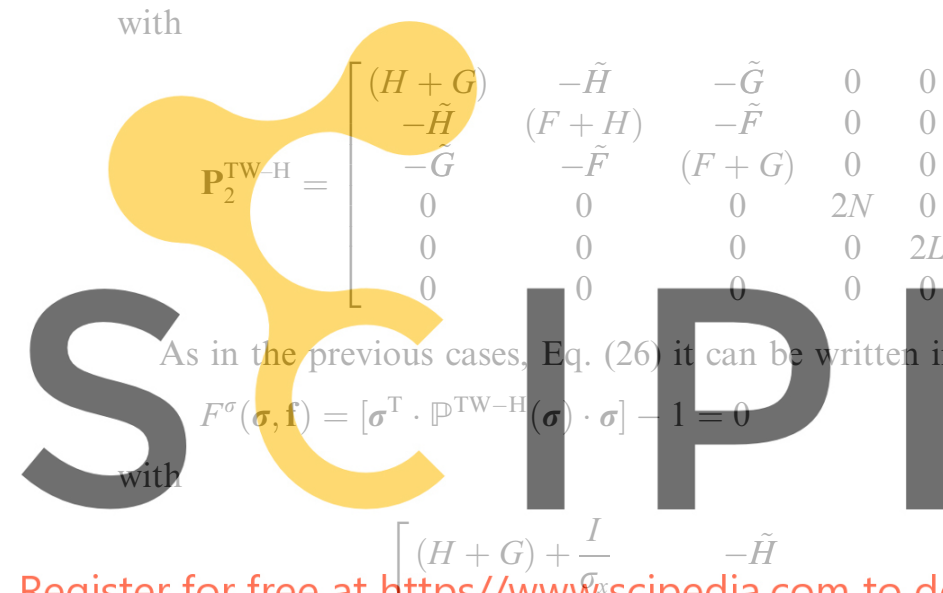

$$
\mathbb{P}^{\mathrm{TW}-\mathrm{H}}(\boldsymbol{\sigma})=\left[\begin{array}{cccccc}
-\tilde{H} & (F+H)+\frac{\sigma_{y}}{\sigma_{y}} & -\tilde{F} & 0 & 0 & 0 \\
-\tilde{G} & -\tilde{F} & (F+G)+\frac{K}{\sigma_{z}} & 0 & 0 & 0 \\
0 & 0 & 0 & 2 N & 0 & 0 \\
0 & 0 & 0 & 0 & 2 L & 0 \\
0 & 0 & 0 & 0 & 0 & 2 M
\end{array}\right],
$$

Register for free at https//www'scipedia.com to download the version without the watermark

where $\boldsymbol{\sigma}^{\mathrm{T}}=\left\{\sigma_{x}, \sigma_{y}, \sigma_{z}, \tau_{x y}, \tau_{y z}, \tau_{x z}\right\}$ is the row-matrix representation of the stress tensor, while $\mathbf{P}_{2}^{\text {ort }}=\mathbf{P}_{2}^{\mathrm{TW}-\mathrm{H}}$ is the square matrix that together with the row matrix $\mathbf{P}_{1}^{\text {ort }}=\mathbf{P}_{1}^{\mathrm{TW}-\mathrm{H}}$ allows the canonical form of the Tsai-Wu and Hoffman criteria expressed in Eq. (26) to be recovered. Also, the square matrix $\mathbb{P}^{\text {ort }}(\boldsymbol{\sigma})=\mathbb{P}^{\mathrm{TW}-\mathrm{H}}(\boldsymbol{\sigma})$ gives an alternative definition of the matrix expression allowing Eq. (26) to be recovered.

Comparing Eqs. (26) and (10), one finds the following relations between the yield stresses and Tsai-Wu/ Hoffman polynomial parameters

$$
\left\{\begin{array}{lll}
(G+H)=\frac{1}{f_{x}^{\mathrm{t}} f_{x}^{\mathrm{c}}}, & 2 L=\frac{1}{f_{y z}^{2}}, \quad I=\frac{1}{f_{x}^{\mathrm{t}}}-\frac{1}{f_{x}^{\mathrm{c}}} \\
(F+H)=\frac{1}{f_{y}^{\mathrm{t}} f_{y}^{\mathrm{c}}}, & 2 M=\frac{1}{f_{x z}^{2}}, \quad J=\frac{1}{f_{y}^{\mathrm{t}}}-\frac{1}{f_{y}^{\mathrm{c}}} \\
(F+G)=\frac{1}{f_{z}^{\mathrm{t}} f_{z}^{\mathrm{c}}}, & 2 N=\frac{1}{f_{x y}^{2}}, \quad K=\frac{1}{f_{z}^{\mathrm{t}}}-\frac{1}{f_{z}^{\mathrm{c}}}
\end{array}\right.
$$


The other matrix coefficients of Eq. (26) are expressed, in complete agreement with Eq. (10), as

- For the Tsai-Wu criterion:

$$
\tilde{F}=\frac{1}{\sqrt{f_{y}^{\mathrm{t}} f_{y}^{\mathrm{c}} f_{z}^{\mathrm{t}} f_{z}^{\mathrm{c}}}}, \quad 2 \tilde{G}=\frac{1}{\sqrt{f_{z}^{\mathrm{t}} f_{z}^{\mathrm{c}} f_{x}^{\mathrm{t}} f_{x}^{\mathrm{c}}}}, \quad 2 \tilde{H}=\frac{1}{\sqrt{f_{x}^{\mathrm{t}} f_{x}^{\mathrm{c}} f_{y}^{\mathrm{t}} f_{y}^{\mathrm{c}}}} .
$$

- For the Hoffman criterion:

$$
2 \tilde{F}=-\frac{1}{f_{x}^{\mathrm{t}} f_{x}^{\mathrm{c}}}+\frac{1}{f_{y}^{\mathrm{t}} f_{y}^{\mathrm{c}}}+\frac{1}{f_{z}^{\mathrm{t}} f_{z}^{\mathrm{c}}}, \quad 2 \tilde{G}=-\frac{1}{f_{y}^{\mathrm{t}} f_{y}^{\mathrm{c}}}+\frac{1}{f_{z}^{\mathrm{t}} f_{z}^{\mathrm{c}}}+\frac{1}{f_{x}^{\mathrm{t}} f_{x}^{\mathrm{c}}}, \quad 2 \tilde{H}=-\frac{1}{f_{z}^{\mathrm{t}} f_{z}^{\mathrm{c}}}+\frac{1}{f_{x}^{\mathrm{t}} f_{x}^{\mathrm{c}}}+\frac{1}{f_{y}^{\mathrm{t}} f_{y}^{\mathrm{c}}} .
$$

As another verification, substituting the equal yield stresses in tension and compression in all orthogonal directions $\left(f_{i} \equiv f_{i}^{\mathrm{c}}=f_{i}^{\mathrm{t}} \forall i=x, y, z\right.$, which gives $I=J=K=0$ or $\left.\mathbf{P}_{1}^{\mathrm{TW}-\mathrm{H}}=\mathbf{0}\right)$ into Eqs. (31)-(33) recovers the Mises-Hill parameters given by Eqs. (20) and (22).

\section{Implicit general definition of an orthotropic yield criterion}

\section{The aim of the implicit general definition of an orthotropic yield criterion is to use an isotropic formulation} in a fictitious space and then transform it into an implicit orthotropic formulation in a real space. This means that the mathematical form of this orthotropic criterion need not be expressed explicitly as shown in Eq. (10), or in the traditional form as shown in the references $[1,2,8,10,22]$; it is sufficient to express its isotropic form explicitly and to assume the existence of a numerical transformation that allows a passage from an isotropic criterion to an imp ic This implicit formulation

\section{tensor $[4,21]$ or mapped-tent} Appendix A). It is based (with elements designated $\sigma$ ), in which the implicit orthotropic yiet
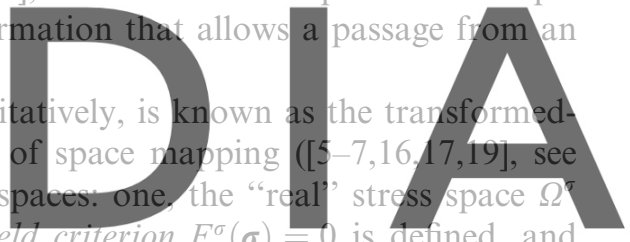

another, the "fictitious" stress space $\bar{\Omega}^{\sigma}$ (with elements designated $\bar{\sigma}$ ), in which the explicit isotropic vield

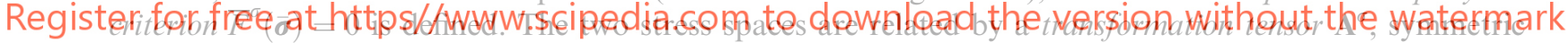

and of rank four, which allows a one-to-one mapping of an image of the stress tensor defined in one space into the other and vice versa. That is,

$$
\overline{\boldsymbol{\sigma}} \stackrel{\text { def }}{=} \mathbf{A}^{\sigma}: \boldsymbol{\sigma} \quad \text { or } \quad \bar{\sigma}_{i j} \stackrel{\text { def }}{=} A_{i j k l}^{\sigma} \sigma_{k l} \text {. }
$$

The fourth-rank tensor $\mathbf{A}^{\sigma} \equiv A_{i j k l}^{\sigma}$ embodies the orthotropic yield behavior, and is sufficient for approximating an explicit isotropic function to any proposed implicit orthotropic function.

The definition of the implicit orthotropic yield criterion results from assuming that in the fictitious stress space there exists an isotropic yield criterion of the form $\bar{F}^{\sigma}(\overline{\boldsymbol{\sigma}}, \mathbf{f})=\left[\overline{\boldsymbol{\sigma}}^{\mathrm{T}} \cdot \mathbf{P}_{2}^{\text {iso }} \cdot \overline{\boldsymbol{\sigma}}+\mathbf{P}_{1}^{\text {iso }} \cdot \overline{\boldsymbol{\sigma}}\right]-1=0$, equivalent to the one defined in Eq. (2), and which is the image of the orthotropic yield criterion whose approximation is sought. This assertion will be proved in what follows by substituting in the orthotropic yield criterion the tensor transformation (34), resulting in the orthotropic yield criterion in the fictitious isotropic space

$$
\left\{\begin{array}{l}
\text { (a) Orthotropic yield criterion in the real space: } \\
F^{\sigma}(\boldsymbol{\sigma}, \mathbf{f})=\left[\boldsymbol{\sigma}^{\mathrm{T}} \cdot \mathbf{P}_{2}^{\text {ort }} \cdot \boldsymbol{\sigma}+\mathbf{P}_{1}^{\text {ort }} \cdot \boldsymbol{\sigma}\right]-1=0, \\
F^{\sigma}(\boldsymbol{\sigma}, \mathbf{f}) \equiv F^{\sigma}\left(\overline{\boldsymbol{\sigma}}, \mathbf{A}^{\sigma}, \mathbf{f}\right)=\left[\left(\mathbf{A}^{\sigma}\right)^{-1} \cdot \overline{\boldsymbol{\sigma}}\right]^{\mathrm{T}} \cdot \mathbf{P}_{2}^{\text {ort }} \cdot\left[\left(\mathbf{A}^{\sigma}\right)^{-1} \overline{\boldsymbol{\sigma}}\right]+\mathbf{P}_{1}^{\text {ort }} \cdot\left[\left(\mathbf{A}^{\sigma}\right)^{-1} \overline{\boldsymbol{\sigma}}\right]-1=0, \\
F^{\sigma}\left(\overline{\boldsymbol{\sigma}}, \mathbf{A}^{\sigma}, \mathbf{f}\right)=\overline{\boldsymbol{\sigma}}^{\mathrm{T}} \cdot \underbrace{\left[\left(\mathbf{A}^{\sigma}\right)^{-\mathrm{T}} \cdot \mathbf{P}_{2}^{\text {ort }} \cdot\left(\mathbf{A}^{\sigma}\right)^{-1}\right]}_{\mathbf{P}_{2}^{\text {iso }}} \cdot \overline{\boldsymbol{\sigma}}+\underbrace{\left[\mathbf{P}_{1}^{\text {ort }} \cdot\left(\mathbf{A}^{\sigma}\right)^{-1}\right]}_{\mathbf{P}_{1}^{\text {iso }}} \cdot \overline{\boldsymbol{\sigma}}-1=0 .
\end{array}\right.
$$

(b) Orthotropic yield criterion in the fictitious isotropic space:

$$
\bar{F}^{\sigma}(\overline{\boldsymbol{\sigma}}, \mathbf{f})=\left[\overline{\boldsymbol{\sigma}}^{\mathrm{T}} \cdot \mathbf{P}_{2}^{\text {iso }} \cdot \overline{\boldsymbol{\sigma}}+\mathbf{P}_{1}^{\text {iso }} \cdot \overline{\boldsymbol{\sigma}}\right]-1=0 \text {. }
$$


From this equation we can derive the tensor $\mathbf{A}^{\sigma}$ that generates the relation $\mathbf{P}_{2}^{\text {ort }}=\left(\mathbf{A}^{\sigma}\right)^{\mathrm{t}} \cdot \mathbf{P}_{2}^{\text {iso }} \cdot\left(\mathbf{A}^{\sigma}\right)$ and $\mathbf{P}_{1}^{\text {ort }}=\mathbf{P}_{1}^{\text {iso }} \cdot\left(\mathbf{A}^{\sigma}\right)$ between the orthotropic matrices $\mathbf{P}_{2}^{\text {ort }}, \mathbf{P}_{1}^{\text {ort }}$ (defining the implicit orthotropic criterion whose approximation is sought, Eq. (35a)) and the isotropic matrices $\mathbf{P}_{2}^{\text {iso }}, \mathbf{P}_{1}^{\text {iso }}$ (defining the fictitious isotropic criterion adopted). Thus, once we choose the isotropic yield criterion $\bar{F}^{\sigma}(\overline{\boldsymbol{\sigma}}, \mathbf{f})=\left[\overline{\boldsymbol{\sigma}}^{\mathrm{T}} \cdot \mathbf{P}_{2}^{\text {iso }} \cdot \overline{\boldsymbol{\sigma}}+\right.$ $\left.\mathbf{P}_{1}^{\text {iso }} \cdot \overline{\boldsymbol{\sigma}}\right]-1=0$ that serves as the basis of the derivation of the implicit orthotropic criterion $F^{\sigma}(\boldsymbol{\sigma}, \mathbf{f})=$ $\left[\boldsymbol{\sigma}^{\mathrm{T}} \cdot \mathbf{P}_{2}^{\text {ort }} \cdot \boldsymbol{\sigma}+\mathbf{P}_{1}^{\text {ort }} \cdot \boldsymbol{\sigma}\right]-1=0$, all that is left to determine is the method of deriving the transformation tensor $\mathbf{A}^{\sigma}$, which will be done in next section.

\section{Definition of the transformation tensor $A^{\sigma}$}

There are several ways of defining the transformation tensor $\mathbf{A}^{\sigma}$, examples of which can be seen in the work of Betten [3], Oller et al. [16,18,19], Car et al. [5-7], and others. Although with these definitions it is possible to find adequate orthotropic yield criteria, it is difficult to adjust them "exactly" to represent desired material behavior. In order to circumvent this limitation, in what follows a new definition of the tensor $\mathbf{A}^{\sigma}$ will be formulated, allowing the exact adjustment of an isotropic criterion to any orthotropic one, by means of the transformed-tensor method (see Appendix A). This is achieved by means of the following relation:

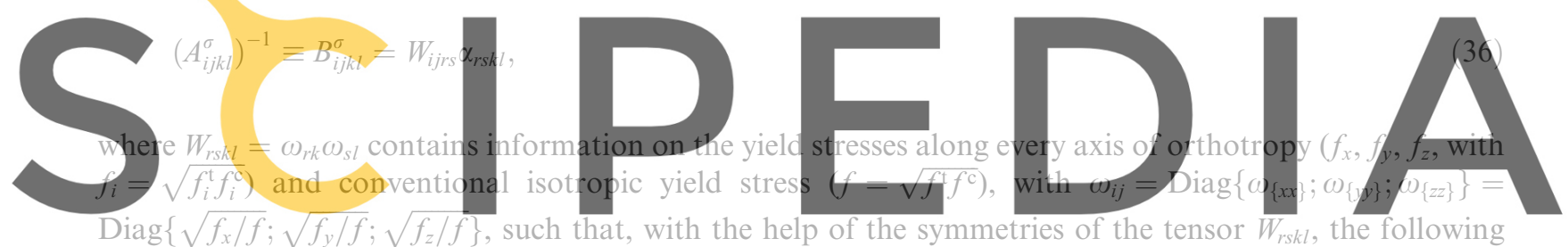

Registertifor ffree at athittps//www.scipedia.com to download the version without the watermark

$$
\begin{aligned}
& W_{i j k l}=\omega_{i k} \omega_{j l}=\left[\begin{array}{cccccc}
\omega_{\{x x\}} \omega_{\{x x\}} & 0 & 0 & 0 & 0 & 0 \\
0 & \omega_{\{y y\}} \omega_{\{y y\}} & 0 & 0 & 0 & 0 \\
0 & 0 & \omega_{\{z z\}} \omega_{\{z z\}} & 0 & 0 & 0 \\
0 & 0 & 0 & \omega_{\{x x\}} \omega_{\{y y\}} & 0 & 0 \\
0 & 0 & 0 & 0 & \omega_{\{y y\}} \omega_{\{z z\}} & 0 \\
0 & 0 & 0 & 0 & 0 & \omega_{\{z z\}} \omega_{\{x x\}}
\end{array}\right] \\
& \rightarrow W_{I J}=\omega_{i i} \omega_{j j}=\left[\begin{array}{cccccc}
\omega_{11} & 0 & 0 & 0 & 0 & 0 \\
0 & \omega_{22} & 0 & 0 & 0 & 0 \\
0 & 0 & \omega_{33} & 0 & 0 & 0 \\
0 & 0 & 0 & \omega_{44} & 0 & 0 \\
0 & 0 & 0 & 0 & \omega_{55} & 0 \\
0 & 0 & 0 & 0 & 0 & \omega_{66}
\end{array}\right] .
\end{aligned}
$$


The tensor $\alpha_{i j k l}$, whose purpose is to adjust $W_{r s k l}$ tensor to the proposed orthotropic yield function, is shown in the following matrix representation:

$$
\begin{gathered}
\alpha_{i j k l}=\left[\begin{array}{cccccc}
\alpha_{\{x x\}\{x x\}} & \alpha_{\{x x\}\{y y\}} & \alpha_{\{x x\}\{z z} & 0 & 0 & 0 \\
\alpha_{\{y y\}\{x x\}} & \alpha_{\{y y\}\{y y\}} & \alpha_{\{y y\}\{z z} & 0 & 0 & 0 \\
\alpha_{\{z z\}\{x x\}} & \alpha_{\{z z\}\{y\}\}} & \alpha_{\{z z\}\{z\}} & 0 & 0 & 0 \\
0 & 0 & 0 & \alpha_{\{x y\}\{x y\}} & 0 & 0 \\
0 & 0 & 0 & 0 & \alpha_{\{y z\}\{y z\}} & 0 \\
0 & 0 & 0 & 0 & 0 & \alpha_{\{z x\}\{z x\}}
\end{array}\right] \\
\rightarrow \alpha_{I J}=\left[\begin{array}{cccccc}
\alpha_{11} & \alpha_{12} & \alpha_{13} & 0 & 0 & 0 \\
\alpha_{21} & \alpha_{22} & \alpha_{23} & 0 & 0 & 0 \\
\alpha_{31} & \alpha_{32} & \alpha_{33} & 0 & 0 & 0 \\
0 & 0 & 0 & \alpha_{44} & 0 & 0 \\
0 & 0 & 0 & 0 & \alpha_{55} & 0 \\
0 & 0 & 0 & 0 & 0 & \alpha_{66}
\end{array}\right]
\end{gathered}
$$

The form of the tensor $B_{i j k l}^{\sigma}=\mathbb{B}^{\sigma} \equiv\left(\mathbf{A}^{\sigma}\right)^{-1}$ with its symmetries is shown in the following matrix repre-
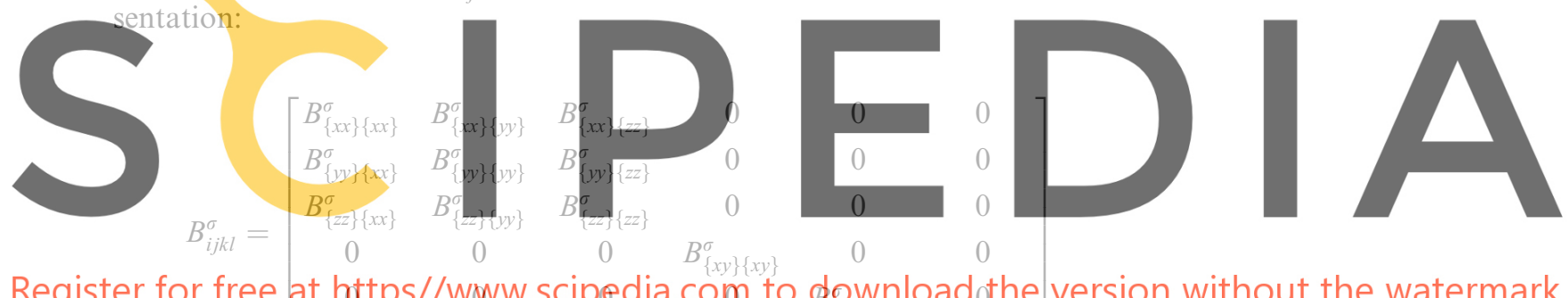

Register for free at https//woww.scipedia.com to gownloadothe version without the watermark

$$
\rightarrow B_{I J}^{\sigma}=\left[\begin{array}{cccccc}
B_{11}^{\sigma} & B_{12}^{\sigma} & B_{13}^{\sigma} & 0 & 0 & 0 \\
B_{21}^{\sigma} & B_{22}^{\sigma} & B_{23}^{\sigma} & 0 & 0 & 0 \\
B_{31}^{\sigma} & B_{32}^{\sigma} & B_{33}^{\sigma} & 0 & 0 & 0 \\
0 & 0 & 0 & B_{44}^{\sigma} & 0 & 0 \\
0 & 0 & 0 & 0 & B_{55}^{\sigma} & 0 \\
0 & 0 & 0 & 0 & 0 & B_{66}^{\sigma}
\end{array}\right] \text { with } B_{12}^{\sigma}=B_{21}^{\sigma}, \quad B_{13}^{\sigma}=B_{31}^{\sigma}, \quad B_{23}^{\sigma}=B_{32}^{\sigma}
$$

This reduced representation of the tensors $\mathbf{B}^{\sigma}, \alpha$ and $\mathbf{W}$, taking advantage of their symmetries, allows the transformation tensor (Eq. (36)) to be derived more simply in the matrix form

$$
\left(A_{I J}^{\sigma}\right)^{-1} \equiv B_{I J}^{\sigma}=W_{I K} \alpha_{K J} \quad \text { or } \quad\left(\mathbf{A}^{\sigma}\right)^{-1} \equiv \mathbf{B}^{\sigma}=\mathbf{W} \cdot \alpha
$$

The isotropic tensor $\mathbf{A}^{\sigma}$ is recovered by enforcing the equality of the uniaxial yield stresses in all directions, $f_{x}=f_{y}=f_{z}=f$, so that the tensor $\omega_{i j} \equiv \delta_{i j}$ coincides with the Kronecker delta, and the matrix form of the adjustment tensor $\alpha_{I J}=I_{I J}=\mathbf{I}$ also coincides with the identity of second rank. This assertion will be confirmed in the next section. 


\section{Numerical derivation of the matrix form of the tensor $B^{\sigma}$}

The procedure for deriving the tensor $\mathbf{B}^{\sigma} \equiv\left(\mathbf{A}^{\sigma}\right)^{-1}$ is based on enforcing the satisfaction of the equality expressed by Eq. (35), that is

$$
\begin{aligned}
& R \equiv\left\{F^{\sigma}(\boldsymbol{\sigma}, \mathbf{f})\right\}-\left\{\bar{F}^{\sigma}(\overline{\boldsymbol{\sigma}}, \mathbf{f})\right\}=0, \\
& R \equiv\left\{\overline{\boldsymbol{\sigma}}^{\mathrm{T}} \cdot\left[\left(\mathbf{A}^{\sigma}\right)^{-\mathrm{T}} \cdot \mathbf{P}_{2}^{\text {ort }} \cdot\left(\mathbf{A}^{\sigma}\right)^{-1}\right] \cdot \overline{\boldsymbol{\sigma}}+\left[\mathbf{P}_{1}^{\text {ort }} \cdot\left(\mathbf{A}^{\sigma}\right)^{-1}\right] \cdot \overline{\boldsymbol{\sigma}}-1\right\}-\left\{\left[\overline{\boldsymbol{\sigma}}^{\mathrm{T}} \cdot \mathbf{P}_{2}^{\text {iso }} \cdot \overline{\boldsymbol{\sigma}}+\mathbf{P}_{1}^{\text {iso }} \cdot \overline{\boldsymbol{\sigma}}\right]-1\right\}=0, \\
& R \equiv \overline{\boldsymbol{\sigma}}^{\mathrm{T}} \cdot\left[\left(\mathbf{B}^{\sigma}\right)^{\mathrm{T}} \cdot \mathbf{P}_{2}^{\text {ort }} \cdot\left(\mathbf{B}^{\sigma}\right)-\mathbf{P}_{2}^{\text {iso }}\right] \cdot \overline{\boldsymbol{\sigma}}+\left[\mathbf{P}_{1}^{\text {ort }} \cdot\left(\mathbf{B}^{\sigma}\right)-\mathbf{P}_{1}^{\text {iso }}\right] \cdot \overline{\boldsymbol{\sigma}}=0 .
\end{aligned}
$$

Taking into account the forms of the isotropic and orthotropic yield criteria expressed by Eqs. (4) and (13), the preceding Eq. (41), which expresses the differences between the real orthotropic yield criterion with the fictitious isotropic one, can also be written as

$$
\begin{aligned}
& R \equiv\left\{F^{\sigma}(\boldsymbol{\sigma}, \mathbf{f})\right\}-\left\{\bar{F}^{\sigma}(\overline{\boldsymbol{\sigma}}, \mathbf{f})\right\}=0, \\
& R \equiv \overline{\boldsymbol{\sigma}}^{\mathrm{T}} \cdot\left[\left(\mathbf{B}^{\sigma}\right)^{\mathrm{T}} \cdot \mathbb{P}^{\mathrm{ort}}(\boldsymbol{\sigma}) \cdot\left(\mathbf{B}^{\sigma}\right)-\mathbb{P}^{\mathrm{iso}}(\boldsymbol{\sigma})\right] \cdot \overline{\boldsymbol{\sigma}}=0 .
\end{aligned}
$$

The non-trivial solution of this problem, for $\overline{\boldsymbol{\sigma}} \neq \mathbf{0}$, is thus reduced to solving the following system of quadratic equations:

$$
\mathbf{R} \equiv\left(\mathbf{B}^{\sigma}\right)^{\mathrm{t}} \cdot \mathbb{P}^{\text {ort }}(\boldsymbol{\sigma}) \cdot\left(\mathbf{B}^{\sigma}\right)-\mathbb{P}^{\text {iso }}(\boldsymbol{\sigma})=\mathbf{0} .
$$

The solution of this system of quadratic equations in $B_{i j k l}^{\sigma} \equiv A_{i j k l}^{\sigma-1}$ produces the symmetric part of this tensor, given by $B_{I J}^{\sigma}$. This in turn yields the transformation tensor $\mathbf{A}^{\sigma}$, which yields an orthotropic implicit yield criterion $F^{\sigma}(\boldsymbol{\sigma}, \mathbf{f})=\left[\boldsymbol{\sigma}^{\mathrm{T}} \cdot \mathbf{P}_{2}^{\text {ort }} \cdot \boldsymbol{\sigma}+\mathbf{P}_{1}^{\text {ort }} \cdot \boldsymbol{\sigma}\right]-1=0$ that coincides exactly with the desired orthotropic criterion $\bar{F}^{\sigma}(\overline{\boldsymbol{\sigma}}, \mathbf{f})=\left[\overline{\boldsymbol{\sigma}}^{\mathrm{T}} \cdot \mathbf{P}_{2}^{\text {iso }} \cdot \overline{\boldsymbol{\sigma}}+\mathbf{P}_{1}^{\text {iso }} \cdot \overline{\boldsymbol{\sigma}}\right]-1=0$ defined in the isotropic space (see Eq. (35)).

The analytic solution of Eq. (43) may be quite difficult, depending on the orthotropic yield criterion that one wishes to approximate, which is why it is more appropriate to attack the solution numerically. The solution of this nonlinear system of equations in $\mathbf{B}^{\sigma}$ is obtained by applying the Newton-Raphson method [20] to the constraint Eq. (43), written in the following form

$$
R_{I J}=\left[\left(B_{K I}^{\sigma}\right)^{\mathrm{t}} \mathbb{P}_{K R}^{\text {ort }}\left(B_{R J}^{\sigma}\right)\right]-\mathbb{P}_{I J}^{\text {iso }}=0,
$$

where $R_{I J}$ is a symmetric matrix representing the residual to be eliminated by means of the NewtonRaphson method. The requirement that the residual $\mathbf{R}$ be reduced to zero can be met by linearizing it in the neighborhood of the current solution at each Newton-Raphson iteration. At the $(i+1)$ th iteration, say, the condition is expressed by means of a Taylor series truncated to its first variation,

$$
\begin{aligned}
& \mathbf{0}=\mathbf{R}^{i+1} \cong \mathbf{R}^{i}+\left[\frac{\partial \mathbf{R}}{\partial \mathbf{B}^{\sigma}}\right]^{i} \cdot\left(\Delta \mathbf{B}^{\sigma}\right)^{i+1} \\
& 0=R_{I J}^{i+1} \cong R_{I J}^{i}+\underbrace{\left[\frac{\partial R_{I J}}{\partial B_{U V}^{\sigma}}\right]^{i}}_{J_{I J U V}^{i}} \cdot\left(\Delta B_{U V}^{\sigma}\right)^{i+1},
\end{aligned}
$$

where $J_{I J U V}$ represents the fourth-order jacobian operator, which allows us to obtain the current value of the unknown by means of the next updating,

$$
\left(B_{U V}^{\sigma}\right)^{i+1}=\left(B_{U V}^{\sigma}\right)^{i}+\left(\Delta B_{U V}^{\sigma}\right)^{i+1} .
$$

This will be the sought-for solution at the $(i+1)$ th iteration if and only if the residual $\mathbf{R}$ satisfies the condition imposed by Eq. (44). However, the nonlinear solution of this quadratic system of equations is not easy task and in some cases it is necessary to reinforce the search of the solution by means of the line search technique. 
Despite the simplifications on the matrix level introduced in Eqs. (37)-(40), the solution of Eq. (44) remains a highly complex task, since it requires the inversion of the fourth-rank jacobian tensor $\left\{J_{I J U V}\right\}^{-1}$. A simple way of solving this system of equations consists of representing the second-order tensors $\mathbf{R}$ and $\mathbf{B}^{\sigma}$ as column matrices using the symmetry properties due to orthotropy, so that the jacobian operator $J_{I J U V}$ reduces to a square matrix $\hat{J}_{I J}$. This simplification on the operational level, helping in the nonlinear solution of the quadratic system of equations, is detailed in the simplified solution algorithm shown below (Table 1), in which $\hat{R}_{I}$ is a column matrix used to represent the residual $R_{I J}$ in this Newton-Raphson algorithm, that is,

$$
R_{I J}=\left[\begin{array}{cccccc}
R_{11} & R_{12} & R_{13} & 0 & 0 & 0 \\
R_{21} & R_{22} & R_{23} & 0 & 0 & 0 \\
R_{31} & R_{32} & R_{33} & 0 & 0 & 0 \\
0 & 0 & 0 & R_{44} & 0 & 0 \\
0 & 0 & 0 & 0 & R_{55} & 0 \\
0 & 0 & 0 & 0 & 0 & R_{66}
\end{array}\right] \Rightarrow \hat{R}_{I}=\left\{\begin{array}{c}
R_{11} \\
R_{12} \\
\vdots \\
R_{33} \\
\vdots \\
R_{66}
\end{array}\right\} .
$$

The unknown is also represented as a column matrix, namely,

$$
B_{I J}^{\sigma}=\left[\begin{array}{cccccc}
B_{11}^{\sigma} & B_{12}^{\sigma} & B_{13}^{\sigma} & 0 & 0 & 0 \\
B_{21}^{\sigma} & B_{22}^{\sigma} & B_{23}^{\sigma} & 0 & 0 & 0 \\
B_{31}^{\sigma} & B_{32}^{\sigma} & B_{33}^{\sigma} & 0 & 0 & 0 \\
0 & 0 & 0 & B_{44}^{\sigma} & 0 & 0 \\
0 & 0 & 0 & 0 & B_{55}^{\sigma} & 0 \\
0 & 0 & 0 & 0 & 0 & B_{66}^{\sigma}
\end{array}\right] \Rightarrow \hat{B}_{I}^{\sigma}=\left\{\begin{array}{c}
B_{11}^{\sigma} \\
B_{12}^{\sigma} \\
\vdots \\
B_{33}^{\sigma} \\
\vdots \\
B_{66}^{\sigma}
\end{array}\right\}
$$

\section{START}

1. Reading of initial data.

2. Set up of initial values for: $i=0$ and $\left(\hat{B}_{I}^{\sigma}\right)^{1}$

3. Continue $(i=1+1)$

4. Evaluation of matrix: $\left(\mathbb{P}_{K R}^{\text {ort }}\right)^{i}$ and $\left(\mathbb{P}_{I J}^{\text {iso }}\right)^{i}$

5. Setting: $\left(\hat{B}_{I}^{\sigma}\right)^{i} \Rightarrow\left(B_{I J}^{\sigma}\right)^{i}$

6. Computation of $\left(R_{I J}\right)^{i}=\left[\left(B_{K I}^{\sigma}\right)^{\mathrm{T}} \mathbb{P}_{K R}^{\text {ort }}\left(B_{R J}^{\sigma}\right)\right]^{i}-\left(\mathbb{P}_{I J}^{\text {iso }}\right)^{i} \Rightarrow\left(\hat{R}_{I}\right)^{i}$

7. Check of convergence

If $\sum_{I}\left\|\left(\hat{R}_{I}\right)^{i}\right\|<$ Toler Go To 9

Else $\Rightarrow$ Solution of quadratic equations system

$$
0 \cong\left(\hat{R}_{I}\right)^{i}+\underbrace{\left[\frac{\partial \hat{R}_{I}}{\partial \hat{B}_{J}^{\sigma}}\right]^{i}}_{\left(\hat{J}_{I J}\right)^{i}} \cdot\left(\Delta \hat{B}_{J}^{\sigma}\right)^{i+1} \Rightarrow\left(\Delta \hat{B}_{J}^{\sigma}\right)^{i+1} \cong-\left[\frac{\partial \hat{R}_{I}}{\partial \hat{B}_{J}^{\sigma}}\right]_{i}^{-1} \cdot\left(\hat{R}_{I}\right)^{i}
$$

8. Updating of the unknown value: $\left(\hat{B}_{I}^{\sigma}\right)^{i+1}=\left(\hat{B}_{I}^{\sigma}\right)^{i}+\left(\Delta \hat{B}_{I}^{\sigma}\right)^{i+1}$

9. Go To 3

10. EXIT 
and the jacobian matrix is constructed in a way that is dimensionally compatible with the two preceding definitions, namely

$$
\hat{J}_{I J}=\frac{\partial \hat{R}_{I}}{\partial \hat{B}_{J}^{\sigma}}=\left[\begin{array}{cccc}
\frac{\partial R_{11}}{\partial B_{11}^{\sigma}} & \frac{\partial R_{11}}{\partial B_{12}^{\sigma}} & \cdots & \frac{\partial R_{11}}{\partial B_{66}^{\sigma}} \\
\frac{\partial R_{12}}{\partial B_{11}^{\sigma}} & \frac{\partial R_{12}}{\partial B_{12}^{\sigma}} & \cdots & \frac{\partial R_{12}}{\partial B_{66}^{\sigma}} \\
\vdots & \vdots & \ddots & \vdots \\
\frac{\partial R_{66}}{\partial B_{11}^{\sigma}} & \frac{\partial R_{66}}{\partial B_{12}^{\sigma}} & \cdots & \frac{\partial R_{66}}{\partial B_{66}^{\sigma}}
\end{array}\right] .
$$

\section{Derivation of the orthotropic hill criterion by means of the transformed-tensor method}

As an example, in what follows a comparison will be made, in the plane of the principal stresses $\sigma_{1}$ and $\sigma_{2}$, between the Mises-Hill criterion obtained by means of the transformed-tensor method using isotropic von Mises criterion and its original form. It will be shown that the results are identical. To that end we proceed to the solution of Eq. (43) by the Newton-Raphson method, producing the tensor $\mathbf{B}^{\sigma}=B_{I J}^{\sigma}$, which leads to the formulation of a tensor $\mathbf{A}^{\sigma}$ adjusting the isotropic von Mises criterion to the orthotropic MisesHill criterion by means of the transformed-tensor method (see Appendix A).

Specifically, a hypothetical orthotropic material is assumed to be in a state of plane stress and to have the following yield stresses along the orthotropy axes: $f_{x}=100 \mathrm{MN} / \mathrm{m}^{2}, f_{y}=200 \mathrm{MN} / \mathrm{m}^{2}, f_{z}=100 \mathrm{MN} / \mathrm{m}^{2}$, $f_{x y}=50 \mathrm{MN} / \mathrm{m}^{2}$. The reference isotropic yield stress adopted is $f=100 \mathrm{MN} / \mathrm{m}^{2}$.

With this information we calculate the matrix $\mathbf{P}_{2}^{\text {ort }}=\mathbf{P}_{2}^{\text {Hill }}=\mathbb{P}^{\text {Hill }}$ defining the orthotropic criterion (Eq. (19)), as well as of the matrix $\mathbf{P}_{2}^{\text {iso }}=\mathbf{P}_{2}^{\text {Mises }} \equiv \mathbb{P}^{\text {iso }}={ }^{\text {Mises }}$ defining the chosen isotropic criterion (Eqs. (5) and (8)). Following these preliminary steps the matrix form of the residual $R_{I J}$ (Eq. (44)) is found, and then its rearrangement as the column matrix $\hat{R}_{I}$, in accordance with Eq. (47). In this way the residual $\hat{R}_{I}\left(\mathbf{B}^{\sigma}\right)$ is a function of the $\mathbf{B}^{\sigma}$ tensor, and consequently the jacobian expressed in Eq. (49) can be calculated.

By means of the Newton-Raphson algorithm (Table 1), the following solution is found

$$
\hat{B}_{I}^{\sigma}=\left\{\begin{array}{l}
B_{11}^{\sigma} \\
B_{12}^{\sigma} \\
B_{22}^{\sigma} \\
B_{33}^{\sigma}
\end{array}\right\}=\left\{\begin{array}{c}
-0.75471 \\
1.07702 \\
0.98793 \\
-0.86603
\end{array}\right\} \Rightarrow B_{I J}^{\sigma}=\left[\begin{array}{ccc}
B_{11}^{\sigma} & B_{12}^{\sigma} & 0 \\
B_{21}^{\sigma} & B_{22}^{\sigma} & 0 \\
0 & 0 & B_{33}^{\sigma}
\end{array}\right]=\left[\begin{array}{ccc}
-0.75471 & 1.07702 & 0 \\
1.07702 & 0.98793 & 0 \\
0 & 0 & -0.86603
\end{array}\right] .
$$

With this result substituted appropriately in Eq. (40), the desired transformation tensor is found, expressed as the square matrix

$$
A_{I J}^{\sigma}=\left(B_{I J}^{\sigma}\right)^{-1}=\left[\begin{array}{ccc}
-0.60207 & 0.55227 & 0 \\
0.55227 & 0.4219 & 0 \\
0 & 0 & -1.1547
\end{array}\right] .
$$

The substitution of this tensor in Eq. (35b) produces an implicit orthotropic yield criterion equivalent to the one expressed by Eq. (35a).

Fig. 1 shows, in principal-stress space, the isotropic von Mises and the classical orthotropic Mises-Hill criteria for the yield-stress data given at the beginning of this section. The same figure shows also the adjustment achieved with Eq. (35b) by means of the tensor $\mathrm{A}_{I J}^{\sigma}=\left(B_{J I}^{\sigma}\right)^{-1}=\left(W_{J K} \alpha_{K I}\right)^{-1}$ obtained from Eq. (51). Lastly, the figure also shows the result obtained likewise with Eq. (35b), but using the unadjusted transformation tensor $A_{I J}^{\sigma}=\left(W_{I J}\right)^{-1}$ (Eq. (37)) obtained by means of the standard form of the transformedtensor method as can be found in Betten [3], Oller et al. [16,17,19], Car et al. [5-7]. 


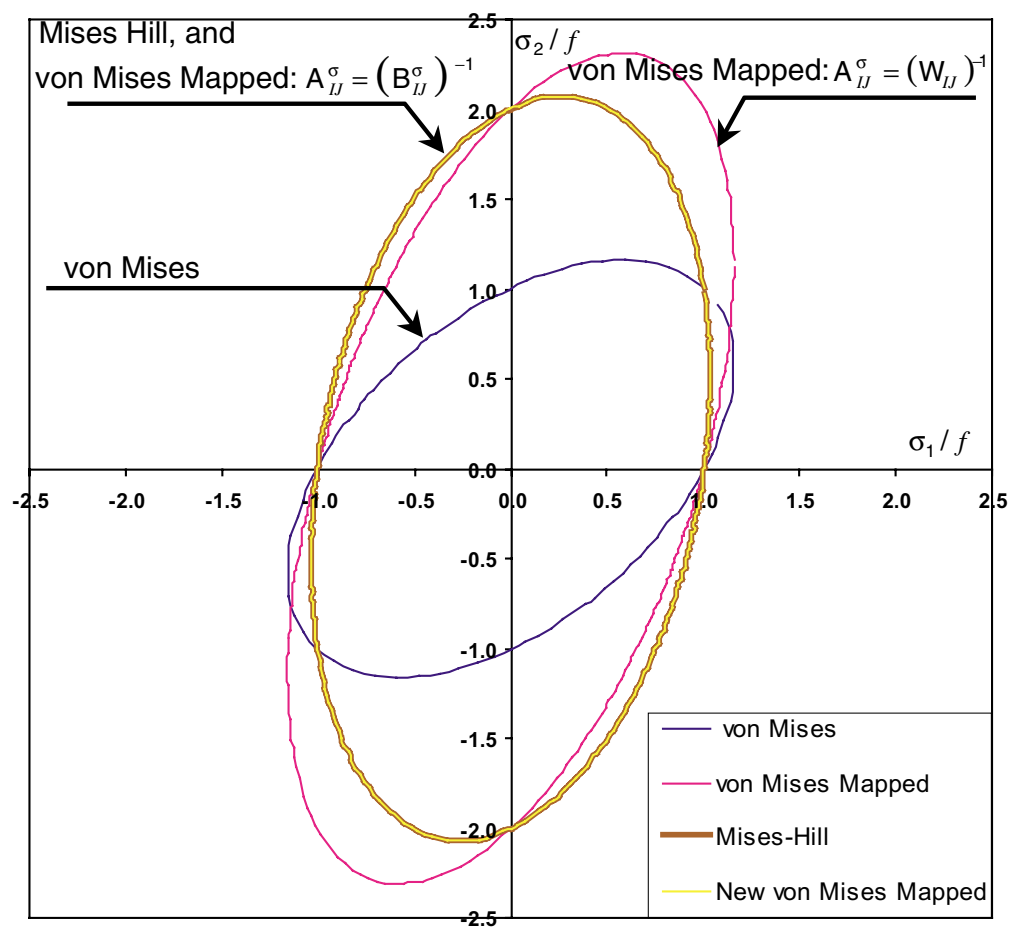

Fig. 1. Representation in $\left(\sigma_{1} / f\right)\left(\sigma_{2} / f\right)$ plane of the isotropic von Mises and the orthotropic Mises-Hill criteria, and of the approximations obtained by the transformed-tensor method, adjusted $A_{I J}^{\sigma}=\left(B_{I J}^{\sigma}\right)^{-1}=\left(W_{I K} \alpha_{K J}\right)^{-1}$ and unadjusted $A_{I J}^{\sigma}=\left(W_{I J}\right)^{-1}$.

\section{Derivation of the orthotropic quadratic Tsai-Wu and Hoffman criteria by means of the transformed-tensor method}

As another example we present a problem in the $\sigma_{1} \sigma_{2}$ plane to show the orthotropic Tsai-Wu [23] and Hoffman [13] criteria obtained by means of the transformed-tensor method using the isotropic DruckerPrager criterion [15]. Again, as the previous example it will be shown that the transformed-tensor method applied to the isotropic Drucker-Prager criterion gives identical results the Tsai-Wu and Hoffman criterion. The principal difference in the procedure with the Mises-Hill/von Mises criterion presented in the previous example is based on the $\mathbf{B}^{\sigma}=\mathbf{B}_{I J}^{\sigma}$ tensor evaluation obtained from Eq. (43) by the Newton-Raphson method. Now, the tensor $\mathbf{A}^{\sigma}$ depends on the stress state at the point at each instant of the mechanical process (see definition of the tensors $\mathbb{P}^{\text {ort }}(\boldsymbol{\sigma})=\mathbb{P}^{\mathrm{TW}-\mathrm{H}}(\boldsymbol{\sigma})$, Eq. (30), and $\mathbb{P}^{\text {iso }}(\boldsymbol{\sigma})=\mathbb{P}^{\text {Drucker }}(\boldsymbol{\sigma})$, Eq. (5)).

A hypothetical orthotropic material is assumed to be in a state of plane stress and to have the following yield stresses along the orthotropy axes: $f_{x}^{\mathrm{t}}=100 \mathrm{MN} / \mathrm{m}^{2}, f_{x}^{\mathrm{c}}=200 \mathrm{MN} / \mathrm{m}^{2}, f_{y}^{\mathrm{t}}=150 \mathrm{MN} / \mathrm{m}^{2}, f_{y}^{\mathrm{c}}=300$ $\mathrm{MN} / \mathrm{m}^{2}, f_{z}^{\mathrm{t}}=100 \mathrm{MN} / \mathrm{m}^{2}, f_{z}^{\mathrm{c}}=200 \mathrm{MN} / \mathrm{m}^{2}, f_{x y}=57.73 \mathrm{MN} / \mathrm{m}^{2}$. The "adopted" reference isotropic yield stresses are $f^{\mathrm{t}}=100 \mathrm{MN} / \mathrm{m}^{2}$ and $f^{\mathrm{c}}=200 \mathrm{MN} / \mathrm{m}^{2}$.

With the yield stresses defined and the level of the stress at each instant, the matrix $\mathbb{P}^{\text {ort }}(\boldsymbol{\sigma})=\mathbb{P}^{\mathrm{TW}-\mathrm{H}}(\boldsymbol{\sigma})$ defining the orthotropic criterion (Eq. (30)), as well as the matrix $\mathbb{P}^{\text {iso }}(\boldsymbol{\sigma})=\mathbb{P}^{\text {Drucker }}(\boldsymbol{\sigma})$ defining the chosen isotropic criterion (Eq. (5)) are obtained.

As in the previous example, following the Newton-Raphson algorithm (Table 1), the Tsai-Wu and Hoffman criteria are obtained in implicit form by means of the transformed-tensor method applied to the isotropic Drucker-Prager criterion. Fig. 2 shows, in principal-stress space, the isotropic Drucker-Prager 


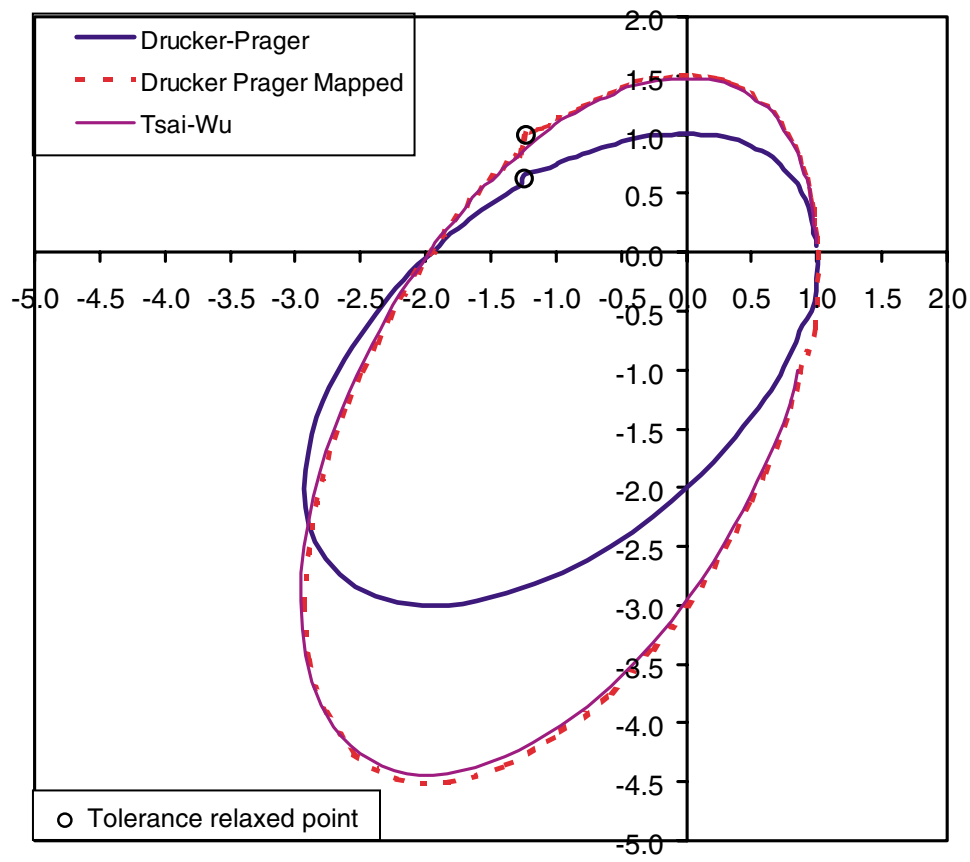

Fig. 2. Representation in $\left(\sigma_{1} / f\right)\left(\sigma_{2} / f\right)$ plane of the isotropic Drucker-Prager and the orthotropic Tsai-Wu criteria, and of the approximations obtained by the transformed-tensor method, adjusted $A_{I J}^{\sigma}=\left(B_{I J}^{\sigma}\right)^{-1}$.

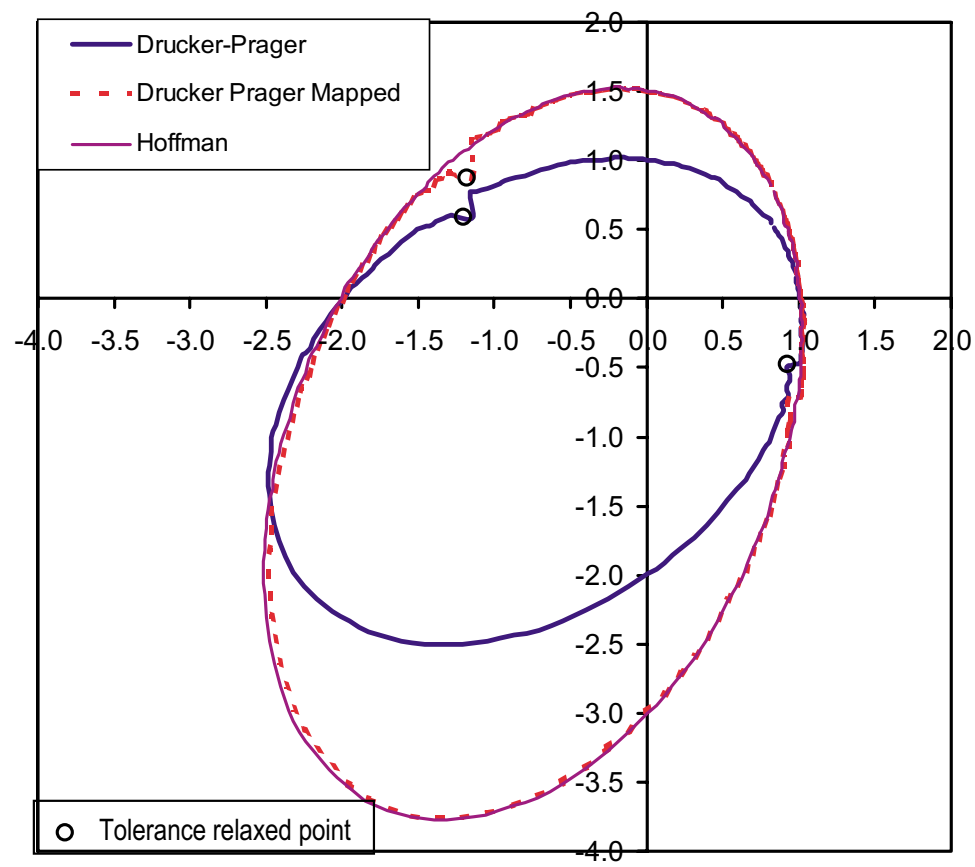

Fig. 3. Representation in $\left(\sigma_{1} / f\right)\left(\sigma_{2} / f\right)$ plane of the isotropic Drucker-Prager and the orthotropic Hoffman criteria, and of the approximations obtained by the transformed-tensor method, adjusted $A_{I J}^{\sigma}=\left(B_{I J}^{\sigma}\right)^{-1}$. 
and the "real orthotropic Tsai-Wu" criteria just above the "implicit Tsai-Wu" criterion mapped by Drucker-Prager. In the same way, Fig. 3 shows, the isotropic Drucker-Prager and the "real orthotropic Hoffman" criteria just above the "implicit Hoffman" criterion mapped by Drucker-Prager too. In both Figs. 2 and 3 the graphic agreement between the "real" and "implicit mapped" criteria can be seen.

The resulting error of the comparison between the "real" criterion versus the "implicit mapped" one reaches a maximum of 3\% at some stress points (see the instability shown in Figs. 2 and 3). This situation is due to the non-linear solution of the quadratic system of Eq. (44) by the Newton-Raphson method (see Table 1), which is usually complicated. At these stress points the convergence tolerances were relaxed in order to obtain the solution. An important point of study in the future will be the improvement of the procedure to solve this system of equations.

\section{Conclusion}

This work presents two generalized ways of expressing an orthotropic yield criterion. The first way is presented as an explicit general function for the orthotropic yield criterion, which can be specialized to produce the Mises-Hill, Tsai-Wu and Hoffman criteria. The second general form is an implicit definition of the orthotropic yield function based on the transformed-tensor method and, as examples of the power of this formulation, the Mises-Hill, Tsai-Wu and Hoffman criteria are shown to be particular implicit formulations obtained from an isotropic yield criterion. The objective of this last formulation is to adjust an arbitrary isotropic yield criterion to the behavior of an anisotropic material.

In view of the good results obtained, both formulations can be useful for the numerical solution of the non-linear behavior of anisotropic solids, allowing a more precise and general adjustment to the real behavior of the solid. Nevertheless, a great deal of work remains to be done, both in the simplification of the formulation and in making the numerical adjustment more efficient.

\section{Acknowledgements}

This work has been supported by the Spanish government through DGICYT under the contract MAT 2000-0741-C02-01. We would also like to thank the Spanish Ministry of Science and Education for the fellowship support given to Prof. Lubliner during his previous stay in Barcelona.

\section{Appendix A. Anisotropic plasticity using the transformed-tensor method}

The transformed-tensor method is based on assuming the existence of a real anisotropic space of stresses $\sigma_{i j}$ and a conjugate space of strains $\varepsilon_{i j}$, such that each of these spaces has its respective image in a fictitious isotropic space of stresses $\bar{\sigma}_{i j}$ and strains $\bar{\varepsilon}_{i j}$, respectively (see Fig. 4), the relation between being defined by

$$
\bar{\sigma}_{i j} \stackrel{\text { def }}{=} A_{i j k l}^{\sigma} \sigma_{k l}, \quad \bar{\varepsilon}_{i j} \stackrel{\text { def }}{=} A_{i j k l}^{\varepsilon} \varepsilon_{k l},
$$

where $A_{i j k l}^{\sigma}$ and $A_{i j k l}^{\varepsilon}$ are the transformation tensors, for stress and strain, respectively, relating the fictitious and real spaces. These fourth-rank tensors embody the natural anisotropic properties of the material.

The stress transformation tensor $A_{i j k l}^{\sigma}$ is a result of the properties of the materials and the shape of the yield surface, as shown Eq. (36),

$$
A_{i j k l}^{\sigma} \equiv\left(B_{i j k l}^{\sigma}\right)^{-1}=\left(W_{i j r s} \alpha_{r s k l}\right)^{-1},
$$




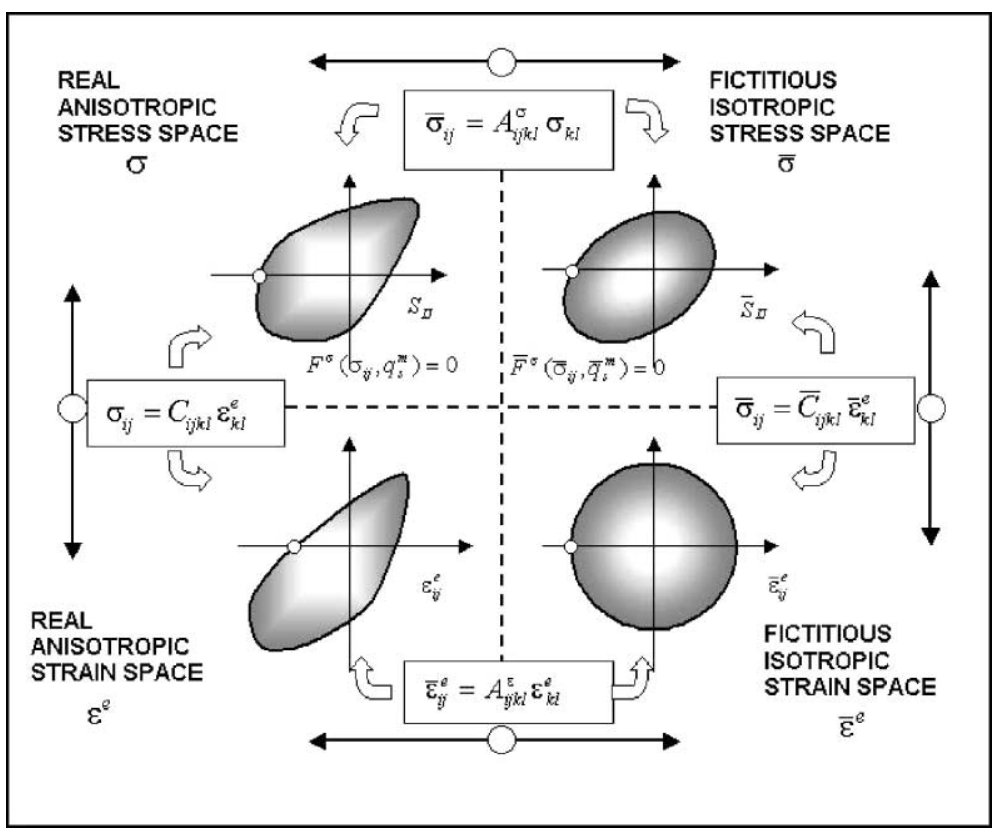

Fig. 4. Relation between the fictitious isotropic and the real anisotropic spaces.

where $W_{i j r s}$ contains information on the yield stresses along every axis of orthotropy, and $\alpha_{r s k l}$ is the shape adjustment tensor (see Section 6).

The relation between the stress and strain transformation tensors can therefore be expressed as $[7,16]$

$$
A_{r s m n}^{\varepsilon}=\left[\bar{C}_{r s i j}^{\sigma}\right]^{-1} A_{i j k l}^{\sigma} C_{k l m n}^{\sigma},
$$

where $\bar{C}_{i j k l}^{\sigma}$ and $C_{i j k l}^{\sigma}$ represent the constitutive tensors in the fictitious and real space, respectively.

This formulation allows the solution for the behavior of a point in the real anisotropic solid by transporting it into a fictitious isotropic space, in which a classical isotropic formulation is used. It is thus possible to use the classical isotropic formulation of small-deformation plasticity for the solution of an anisotropic plasticity problem. In this context, the anisotropic yield function $F^{\sigma}$ and the anisotropic plastic potential $G^{\sigma}$ are defined, respectively, as follows:

$$
F^{\sigma}\left(\sigma_{i j}, q_{s}^{m}\right) \equiv \bar{F}^{\sigma}\left(\bar{\sigma}_{i j}, \bar{q}_{s}^{m}\right)=0, \quad G^{\sigma}\left(\sigma_{i j}, q_{s}^{m}\right) \equiv \bar{G}^{\sigma}\left(\bar{\sigma}_{i j}, \bar{q}_{s}^{m}\right)=K,
$$

where $q_{s}^{m}$ represents the set of $m$ internal variables and $K$ is a constant. The plastic flow rule is defined by the chain rule as [16]

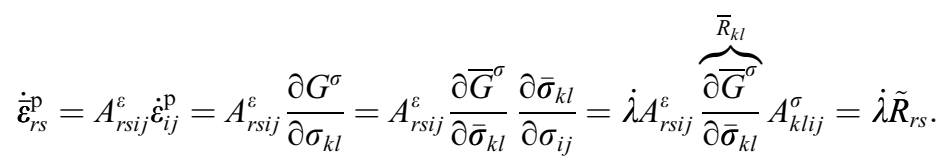

Note that $\bar{R}_{i j}$ is the direction tensor of the classical flow rule in an isotropic space and is modified by $A_{i j k l}^{\sigma}$ in order to obtain its analogue in the anisotropic space, $R_{i j}=\bar{R}_{k l} A_{k l i j}^{\sigma}$. The transformation $\tilde{R}_{r s}=A_{r s i j}^{\varepsilon} R_{i j}$ introduces the influence of anisotropic elasticity on anisotropic yielding. 
The secant and tangent constitutive tensors in real stress space are expressed as follows:

$$
\begin{aligned}
\sigma_{i j} & =m \frac{\partial \Psi^{\sigma}\left(\varepsilon_{k l}^{\mathrm{e}}, q_{s}^{m}\right)}{\partial \varepsilon_{i j}^{\mathrm{e}}}=\left(A_{i j k l}^{\sigma}\right)^{-1} \bar{\sigma}_{k l} \Rightarrow \dot{\sigma}_{i j}=\left(A_{i j k l}^{\sigma}\right)^{-1} \dot{\bar{\sigma}}_{k l} \equiv\left(A_{i j k l}^{\sigma}\right)^{-1}\left(\bar{C}_{k l r s}^{\sigma}\right)^{\mathrm{ep}} \dot{\bar{\varepsilon}}_{r s}\left(\bar{C}_{i j k l}^{\sigma}\right)^{\mathrm{ep}} \\
= & \bar{C}_{i j k l}^{\sigma}-\frac{\left(\bar{C}_{i j k l}^{\sigma} \tilde{R}_{r s}\right)\left(\frac{\partial \bar{F}^{\sigma}}{\partial \bar{\sigma}_{r s}} \bar{C}_{r s k l}^{\sigma}\right)}{H+\frac{\partial \bar{F}^{\sigma}}{\partial \bar{\sigma}_{p q}} \bar{C}_{p q t n}^{\sigma} \tilde{R}_{t n}},
\end{aligned}
$$

where $\Psi^{\sigma}$ is the free-energy density, $m$ is the mass density and $H$ is the hardening parameter.

\section{References}

[1] F. Barlat, J. Lian, Plastic behaviour and stretchability of sheet metals. Part I - a yield function for orthotropic sheets under plane stress conditions, Int J. Plast. 5 (1989) 51-66.

[2] F. Barlat, D.J. Lege, J.C. Brem, A six-component yield function for anisotropic materials, Int. J. Plast. 7 (1991) 693-712.

[3] J. Betten, Creep theory of anisotropic solids, J. Rheol. 25 (6) (1981) 565-581.

[4] J.P. Boehler, A. Sawczuk, Équilibre limite des sols anisotropes, J. Méc. 9 (1970) 5-32.

[5] E. Car, S. Oller, E. Oñate, Numerical constitutive model for laminated composite materials, in: J.A. Covas (Ed.) Second ESAFORM Conference on Material Forming, Minho, Portugal, 1999a, pp. 147-150.

[6] E. Car, S. Oller, E. Oñate, A large strain plasticity model for anisotropic material - composite material application, Int. J. Plast. 17 (2001) 1437-1463.

[7] E. Car, S. Oller, E. Oñate, An anisotropic elastoplastic constitutive model for large strain analysis of fiber reinforced composite materials, Comput. Methods Appl. Mech. Engrg. 185 (2-4) (2000) 245-277.

[8] E. Chu, Generalization of Hill's 1979 anisotropic yield criteria, NUMISHEET 93, Isehara, Japan, 1993 pp. $199-208$.

[9] Decolon, Ch., 2002. Analysis of composite structures, Hermes Penton Ltd.

[10] M. Dutko, D. Péric, D.R.J. Owen, Universal anisotropic yield criterion based on superquadric functional representation: part 1. Algorithmic issues and accuracy analysis, Comp. Methods Appl. Mech. Engrg. 109 (1993) 73-93.

[11] R. Hill, A theory of the yielding and plastic flow of anisotropic metals, Proc. Roy. Soc. Lon. Ser. A. 193 (1984) $281-297$.

[12] R. Hill, Constitutive modeling of orthotropic plasticity in sheet metals, J. Mech. Phys. Solids 38 (3) (1990) $405-417$.

[13] O. Hoffman, The brittle strength of orthotropic materials, J. Comp. Mater. 1 (1967) 200-206.

[14] G.A. Maugin, The thermomechanics of plasticity and fracture, Cambridge University Press, 1992.

[15] J. Lubliner, Plasticity Theory, Macmillan Publishing, USA, 1990.

[16] S. Oller, S. Botello, J. Canet, E. Oñate, An anisotropic elasto-plastic model based on an isotropic formulation, Int. J. Comput. Eng. Comput. 12 (3) (1995) 245-262.

[17] S. Oller, E. Oñate, J. Canet, S. Botello, A plastic damage constitutive model for composite materials, Int. J. Solids Struct. 33 (17) (1996a) 2501-2518.

[18] S. Oller, E. Oñate, J. Canet, A mixing anisotropic formulation for analysis of composites, Commun. Numer. Methods Engrg. 12 (1996b) 471-482.

[19] S. Oller, J. Rubert, Las E. Casas, E. Oñate, S. Proença, Large strains elastoplastic formulation for anisotropic materials, in: J. Chenot, J. Agassant, P. Montnitinnet, B. Bergnes, N. Billon (Eds.), First Esaform Conference on Material Forming, Sophia Antipolis, France, March, pp. 191-194.

[20] W.H. Press, S.A. Teulosky, W.T. Vetterling, B.P. Flannery, Numerical recipes in Fortran 77, in: The Art of Scientific Computing, Volume I, Cambridge University Press, 1992.

[21] Z. Sobotka, Theorie des plastischen Fließens von ainsotropen Körpern, Z. Angew. Math. Mech. 49 (1969) $25-32$.

[22] C. Truesdell, R. Toupin, The classical field theories, in: S. Flügge (Ed.), Handbuch der Physik III/I, Springer-Verlag, Berlin, 1960.

[23] S.W. Tsai, E.M. Wu, A general theory of strength for anisotropic materials, J. Comp. Mater. 5 (1971) 58-80. 